\title{
Comparison of X-ray and neutron tomographic imaging to qualify manufacturing of a fusion divertor tungsten monoblock
}

\author{
LI.M. Evans ${ }^{1,2, *}$, T. Minniti ${ }^{3}$, M. Fursdon ${ }^{1}$, M. Gorley ${ }^{1}$, T. Barrett ${ }^{1}$, F. Domptail ${ }^{1}$, E. Surrey ${ }^{1}$, W. \\ Kockelmann ${ }^{3}$, A. v. Müller ${ }^{4}$, F. Escourbiac ${ }^{5}$, A. Durocher ${ }^{5}$ \\ ${ }^{1}$ Culham Centre for Fusion Energy, Culham Science Centre, Abingdon, Oxon, OX14 3DB, UK \\ ${ }^{2}$ College of Engineering, Swansea University, Bay Campus, Fabian Way, Swansea, SA1 8EN, UK \\ ${ }^{3}$ STFC, Rutherford Appleton Laboratory, ISIS Facility, Harwell, OX11 0QX, UK \\ ${ }^{4}$ Max-Planck-Institut für Plasmaphysik, Boltzmannstraße 2, 85748 Garching, Germany \\ 5ITER Organization, Route de Vinon-sur-Verdon, CS 90 046, 13067 St. Paul Lez Durance Cedex, France
}

\section{Abstract}

Within a tokamak fusion energy device, the performance and lifespan of a divertor monoblock under high heat flux cycles is of particular interest. Key to this is the quality of manufacture, especially the material joining interfaces. Presented here is a comparative study between $\mathrm{X}$-ray and neutron tomography to investigate the quality of manufactured monoblocks. Tungsten is a high attenuator of X-rays, thus X-ray tomography was performed on 'region of interest' samples where the majority of the tungsten armour was removed to reduce the attenuation path. Neutron tomography was performed on the full monoblock samples for non-destructive testing and on the 'region of interest' samples for direct comparison. Both techniques were shown to be capable of imaging the samples but having their own advantages and disadvantages relating to image accuracy and logistical feasibility. The techniques discussed are beneficial for either the research and development cycle of fusion component design or in quality assurance of manufacturing.

\section{Introduction}

Due to its location and function within a tokamak, the divertor is the component subjected to the greatest steady thermal load. During steady-state operation thermal fluxes are expected to be at least $10 \mathrm{MW} \cdot \mathrm{m}^{-2}[1]$. To remain within operational temperature limits the divertor components are actively cooled [2]. This is achieved by connecting armour tiles through their centres to a pipe carrying coolant (coined a monoblock). As the function of this heat sink is to transfer thermal energy away from the armour, it is imperative that the method of joining the armour to the pipe must provide a bond that retains both structural integrity and a high thermal conductivity under large thermal loads. As this region will contribute to, and potentially dominate, performance of the component, it is of utmost importance that the armour-pipe interface is well characterised.

For ITER, the monoblock will use tungsten (W) for the armour with a copper alloy (CuCrZr) cooling pipe. The armour is bonded to the pipe to maintain thermal conduction, but a large thermal expansion coefficient mismatch between the $\mathrm{W}$ and $\mathrm{CuCrZr}$ causes high levels of stress within the part. Therefore, a functional compliant interlayer is used at the material interface to create a bond between the pipe and armour with improved longevity. For future devices, where it would be desirable to operate at higher thermal fluxes, alternatives designs are being investigated e.g. using composite materials, a functionally graded interlayer or geometric constructs [3]. ITER will use approximately 320,000 monoblocks which will require replacing after 5 full power years (fpy) of operation due to degradation [4], therefore manufacturing cost is a consideration. In addition to investigating

\footnotetext{
* Corresponding author. Tel: +44 1792 513625. E-mail: llion.evans@swansea.ac.uk (LI.M. Evans).
} 
alternative designs, various manufacturing routes are being tested which aim to reduce this cost e.g. bonding of the armour to heat sink materials via brazing rather than direct casting of copper.

Because of this, the capability to inspect the material interfaces within the monoblock is of great value. This is true for both quality assurance, when manufacturing the current generation, and informing decisions in the development of next generation monoblocks. The features of concern in this region are anything that may reduce the component's lifespan by reducing its ability to withstand high thermal loads. For example, micro-cracking or voids will act as thermal barriers which can increase peak temperatures or act as crack or interface debonding initiation sites when experiencing thermal fatigue. Deviations from design tolerance cause differences between real and predicted stress and temperature fields which may exceed safety limits. For this component, tolerances of interest are interlayer thicknesses and small-scale geometric constructs. For future designs which may include composite materials, the exact fibre placement or matrix permeation may be of importance due to localised variations in the material's performance leading to stress concentration zones or thermal hot spots. Finally, other features such as material inclusions or the flow of filler material from the brazing process is also of interest. A better understanding of the extent of the existence of these features will aid better informed decisions with regard to the suitability of particular manufacturing routes.

Currently, the main methods for investigating manufacture quality of divertor monoblocks are via conventional optical or scanning electron microscopy (SEM) and ultrasonic scanning. SEM produces nanometre resolution images and may even be used to investigate compositional makeup e.g. elemental diffusion at material interfaces [5]. However, the technique is destructive as the sample must be cut in preparation of imaging. For brittle materials like tungsten this may introduce defects that were not present within the component. Additionally, two dimensional cross-sections showing features like cracks or inclusions provide insufficient data about the size and shape of features. Serial sectioning techniques may be used for additional data for the third dimension [6] but these are extremely time consuming and have relatively low resolution through thickness. Ultrasonic scanning is very effective in providing a relatively quick verification for the quality of bonding for the current generation design of monoblock. This technique scans radially around the coolant pipe by moving a transducer along the thin edges of the monoblock [7]. A drawback of the technique is its inability to distinguish between voids or inclusions as it only measured the changes in acoustic signal from a baseline value. It is also limited in its relatively low millimetre scale resolution and could not be used to investigate fine tolerance deviations. Additionally, geometric constructs or composite fibres in future generation designs will appear as changes in signal, these may be difficult to distinguish between against component defects. A recent development of this technique is to combine ultrasonic scanning and infrared imaging for improved defect detection [8].

A method which has been increasing in its use within an industrial setting is computerised tomography (CT). This has the benefit of providing three dimensional images which give data about features size and shape. This method depends on contrast in signal attenuation which means it is not well adept for interfaces between similar materials, e.g. carbon fibres in a carbon matrix, but can easily distinguish between voids and inclusions and even determine interfaces between differing metals if there is sufficient attenuation contrast [9].

Various $\mathrm{CT}$ techniques use different signals which are appropriate for the medium being imaged e.g. radio signals are used for upper atmosphere studies [10]. For industrial manufacturing the most 
common method is X-ray tomography [11]. Depending on the precise setup this can provide nanometre resolution but is typically on the micron scale for commonly available commercial scanners [9]. The main challenge with using X-ray tomography for imaging of the divertor monoblock is that tungsten is an extremely high attenuator of $X$-rays. Previous work shows use of synchrotron $X$-rays on cylindrical tungsten samples with a diameter of $0.5 \mathrm{~mm}$ and states that this was the achievable limit [12]. This is relatively small in comparison to the proportion of a monoblock that would be required to provide significant data about the material interfaces. However, recent advances in CT hardware offer higher energy X-rays than previously available which may be sufficient to image portions of the monoblock providing significant data.

Other than X-ray CT, neutrons could provide viable CT signal sources that aren't attenuated excessively by tungsten to such a level that impedes imaging. Neutron CT is a relatively immature technique and can only be performed at a handful of facilities globally [13]. Additionally, when the samples interact with the beamline they become activated. Depending on the materials used in the samples the time required for the samples to reduce sufficiently in activity may be prohibitive in the feasibility in their wide-scale use for component qualification. The neutron damage from the beamline will be insignificant compared of in service use and can therefore be disregarded. Depending on the level of detail provided by this technique the benefits could far outweigh the disadvantages.

This paper investigates and compares the advantages and disadvantages of X-ray and neutron CT imaging of current and future generation divertor monoblocks. This includes the quality of the images themselves, detailing characteristics such as resolution and noise, and the logistical feasibility requiring consideration due to steps such as sample preparation.

\section{Sample manufacturing}

For this study three sample types were used: ITER reference monoblock (ITER_MB), Culham Centre for Fusion Energy thermal break concept monoblock (CCFE_MB) and Max-Planck-Institut für Plasmaphysik tungsten fibre / copper matrix coolant pipe (IPP_Wf-Cu), as shown in Figure 1.
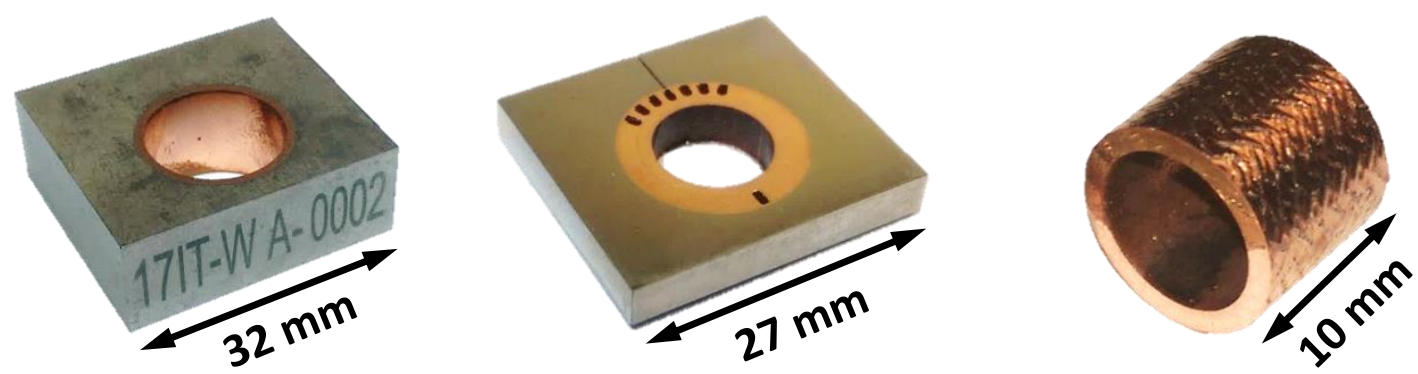

Figure 1. Three sample types used for this work: (left) ITER reference monoblock (ITER_MB), (centre) Culham Centre for Fusion Energy thermal break concept monoblock (CCFE_MB) and (right) Max-Planck-Institut für Plasmaphysik tungsten fibre / copper matrix coolant pipe (IPP_Wf-Cu)

The 'ITER_MB' sample is manufactured by first producing a bar of sintered tungsten which is rolled to yield elongated grains whose longitudinal orientation are aligned such they shall not be parallel to the surface. The tungsten armour is then machined to shape before oxygen free high conductivity (OFHC) copper is directly cast into the internal hole. A drill is then used to machine the copper layer to leave the desired interlayer thickness. For use within the divertor, a series of monoblocks would be placed along a copper alloy ( $\mathrm{CuCrZr}$ ) coolant pipe and joined by hot radial pressing. The main ITER_MB sample 
used in this instance only included the armour and interlayer. A second sample, 'ITER_HHFT', which had been subjected to high heat flux testing prior to imaging also included the coolant pipe.

The 'CCFE_MB' sample was fabricated using a two-stage vacuum braze process. Copper sleeves (for interlayer material) were first brazed to $\mathrm{CuCrZr}$ pipes and the geometric constructs (grooves) machined into the outer surface of the subsequent assembly. Tungsten monoblocks were cast with copper into the central bore (with similar specifications to the ITER_MB sample). This was then machined out to leave a thin copper layer. A stress relieving slit was cut into the plasma facing surface of the tungsten by electro-discharge machining (EDM). A second $\mathrm{Cu}$-to- $\mathrm{Cu}$ braze process was performed where the $\mathrm{W} / \mathrm{Cu}$ blocks are attached to the pipe/sleeve assembly to create the complete mock-up component. The final braze also included a precipitation hardening cycle. The braze filler material used was a 50:50 Au-Cu foil, known commercially as Orobraze 970, which had a thickness of $25 \mu \mathrm{m}$ and was wrapped around the inner part three times before bonding. For more details on the design and manufacture of the monoblock see [14].

For the 'IPP_Wf-Cu' sample, the $W$ fibre preform used was manufactured by means of sequential circular mandrel overbraiding. The raw material used were drawn potassium (K) doped $W$ fibres with a nominal diameter of $150 \mu \mathrm{m}$. The resulting preform comprised 5 braided layers which exhibited a regular 2/2 twill weave repeat pattern. The $\mathrm{W}_{\mathrm{f}} \mathrm{Cu}$ composite material was then produced by infiltrating the braided preform with liquid Cu. Eventually, the infiltrated composite was machined to final dimensions (inner diameter: $10 \mathrm{~mm}$, wall thickness: $1.5 \mathrm{~mm}$ ) and specimens with an axial length of $10 \mathrm{~mm}$ were cut. For further detail on the manufacturing and the motivation for interest in $\mathrm{W}_{\mathrm{f}}-\mathrm{Cu}$ materials for this application see [15].

\section{Methodology}

All radiographic methods (e.g. X-ray or neutron) are based on the same principle: that radiation is attenuated on passing through matter [16]. The specimen is placed in the incident radiation beam. After passing through, the remaining beam enters a detector that registers the fraction of the initial intensity transmitted by each point in the object. A schematic is shown in Figure 2. Inhomogeneities in the object or an internal defect (e.g. void, crack, porosity or inclusion) will show up as a change in radiation intensity reaching the detector. Thus, detection of defects in radiography is based on the observation of differences in radiation intensity after passing through the object under examination. This occurs according to the Beer-Lambert law, see Equation (1), where $I_{0}$ and $I$ are the incident and attenuated intensities, respectively, $\mu$ is the linear attenuation coefficient of the material (combining the interaction cross-section and nuclear density) and $s$ is the path length through the sample.

$$
I(x, y)=I_{0}(x, y) e^{-\int_{p a t h} \mu(x, y, z) d s}
$$

As seen in Equation (1), radiography provides line-integrated information related to the amount of attenuation where contributions from different elements and the amount of material corresponding to the path length cannot be separated. However, in tomography one collects multiple radiographies (projections) for distinct angular orientations. From the tomographic scan, one obtains threedimensional spatially resolved images (i.e. volumetric data), which generally display the attenuation coefficient distribution in the sample volume. This procedure makes use of different reconstruction algorithms, such as filtered back projection (FBP) or iterative based algorithms [16]. 


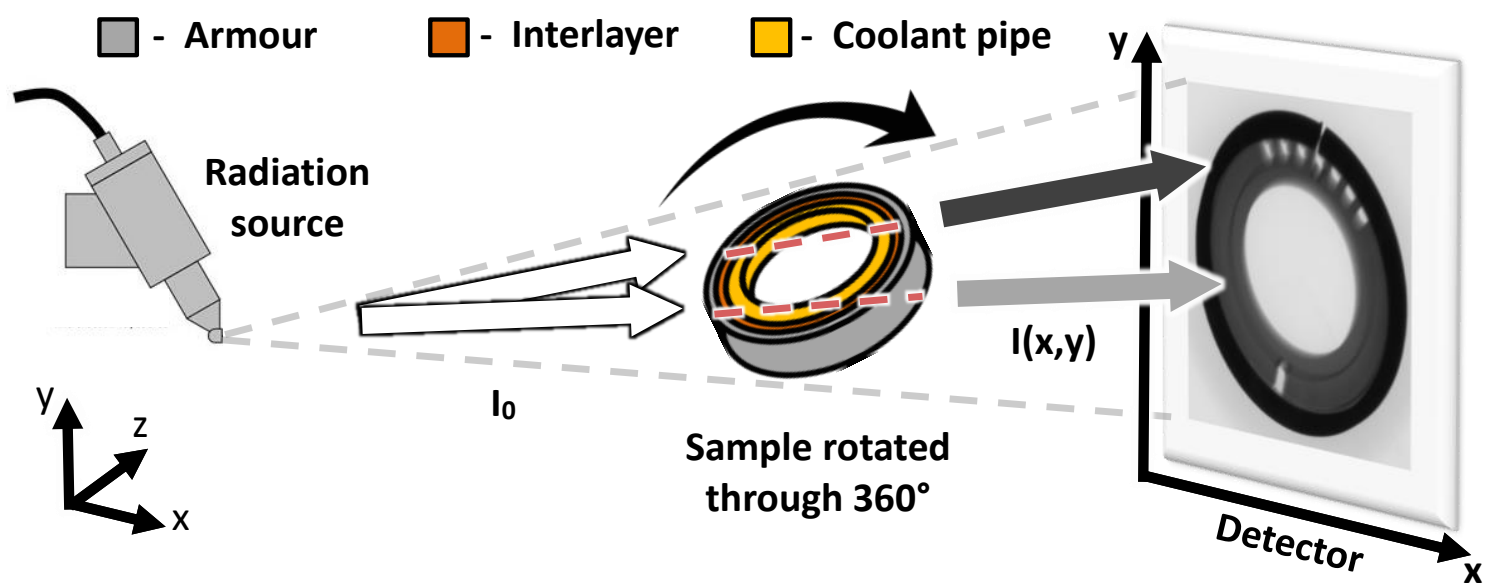

Figure 2. Schematic of X-ray tomography setup. Each detector pixel detector sums the signal attenuation along a particular path. Back projection algorithms use $2 \mathrm{D}$ radiographs taken through $360^{\circ}$ to resolve the attenuation in $3 \mathrm{D}$ space. The main difference with neutron scanning is that the beam is quasi-parallel rather than having a cone angle.

\subsection{X-ray tomography sample preparation and scanning}

Because tungsten is extremely attenuating of X-rays, a sufficiently long attenuation path will reduce the signal such that insufficient data is collected to perform volumetric reconstruction. To reduce the attenuation path length, 'region of interest' (ROI) samples were produced via electro discharge machining (EDM) to remove most of the tungsten. The aim was to leave a sufficient layer to not affect the material interfaces but thin enough to allow X-ray penetration. Samples were visually inspected before and after preparation with optical microscopy. No differences were observed on the external surfaces at the material interfaces. Table 1 and Figure 3 show dimensions and photographs of the resultant $\mathrm{ROI}$ sample, respectively. To further reduce the attenuation path length, the sample was scanned at a $\sim 45^{\circ}$ tilt angle. Industrial X-ray CT is a well-established technique, therefore detailed methodology and hardware specifications can readily be found elsewhere e.g. [17], [18]. Scanning was performed using commercial industrial scanners and followed standard industrial X-ray CT practice. The parameters used for each scan are shown in Table 2. Reconstruction of the 3D volume from 2D radiographs was completed using CT Pro V3.1 (Nikon Metrology NV, Tring, Hertfordshire, UK).

Table 1. Dimensions of region of interest samples.

\begin{tabular}{lccccc}
\hline \multicolumn{1}{c}{ Sample } & $\begin{array}{c}\text { ID/OD } \\
\text { Diameter } \\
(\mathbf{m m})\end{array}$ & $\begin{array}{c}\text { Pipe } \\
\text { thickness } \\
(\mathbf{m m})\end{array}$ & $\begin{array}{c}\text { Tungsten } \\
\text { thickness } \\
\mathbf{( m m})\end{array}$ & $\begin{array}{c}\text { Length } \\
(\mathbf{m m})\end{array}$ & Material \\
\hline ITER_MB_ROI & $15 / 19$ & 2 & 1 & 5 & W-Cu \\
ITER_HHFT_ROI & $12 / 17$ & 2.5 & 0.5 & 3 & W-Cu-CuCrZr \\
CCFE_MB_ROI & $10 / 18$ & 4 & 0.5 & 2 & W-Cu-Cu-CuCrZr \\
IPP_Wf-Cu & $10 / 13$ & 1.5 & 0.15 fibres & 10 & Wf-Cu fibre composite \\
\hline
\end{tabular}
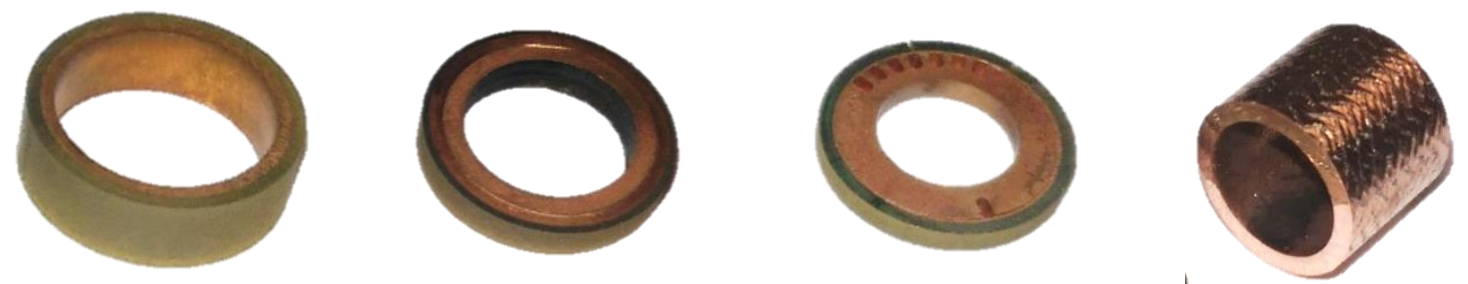

Figure 3. 'Region of interest' sample photographs: a) ITER_MB_ROI, b) ITER_HHFT_ROI, c) CCFE_MB_ROI, d) IPP_Wf-CU. 
Table 2. X-ray tomography scanning parameters.

\begin{tabular}{llllllll}
\hline Sample & Machine & $\begin{array}{l}\text { Voltage } \\
(\mathrm{kV})\end{array}$ & $\begin{array}{l}\text { Current } \\
(\mu \mathrm{A})\end{array}$ & $\begin{array}{l}\text { Filter } \\
(\mathrm{mm})\end{array}$ & $\begin{array}{l}\text { Acquisition } \\
\text { time }(\mathrm{s})\end{array}$ & $\begin{array}{l}\text { Number of } \\
\text { projections }\end{array}$ & $\begin{array}{l}\text { Frames / } \\
\text { projection }\end{array}$ \\
\hline ITER_MB_ROI & NSI X5000 & 240 & 100 & N/A & 500 & 1440 & 3 \\
ITER_HHFT_ROI & NSI X5000 & 240 & 100 & N/A & 500 & 1440 & 3 \\
CCFE_MB_ROI & Nikon 225 & 220 & 80 & $\mathrm{Cu}, 0.5$ & 1000 & 3142 & 1 \\
IPP_Wf-Cu & Nikon 225 & 200 & 220 & $\mathrm{Cu}, 2.0$ & 500 & 2616 & 1 \\
\hline
\end{tabular}

\subsection{Neutron tomography scanning}

The advantage neutron imaging has in comparison to X-ray imaging is that neutrons are significantly more penetrating through tungsten. Thus, it is feasible to image samples containing larger volumes of tungsten. For this work, the importance is that full monoblock samples may be scanned. Neutron tomography was performed at IMAT, ISIS, Rutherford Appleton Laboratory, STFC, UK [19], [20].

In practice the setup for neutron and X-ray tomography is very similar. The sample is placed in between the radiographic source and detector, it is rotated through $360^{\circ}$ whereby a series of $2 \mathrm{D}$ radiographs are taken at uniformly spaced angular steps, as previously shown in Figure 2. Other than the radiographic source, the main difference between these two setups is that neutron imaging uses a quasi-parallel beam rather than a cone angle beam (although a parallel setup is possible for X-ray imaging). Parallel beam geometry is realised in IMAT with a pinhole selector system. Neutrons are forced to propagate, downstream of the neutron guide, through a circular aperture with diameter $D$ before being detected after a distance $L$ by the neutron camera. This produces a specific beam divergence or collimation ratio $L / D$. The sample position at IMAT has a distance $L$ of $10 \mathrm{~m}$. Depending on the specimen characteristic and boundary conditions (neutron flux, spatial resolution of the detector, etc.) such collimation ratio could be changed on IMAT to 125, 250, 500, 1000 and 2000 by changing the pinhole diameter. For more details on IMAT setup see [19]. A large L/D value means a low beam divergence and neutron flux and vice versa [21]. Another important aspect of a parallel beam setup is the maximum distance, $l$, of the object from the imaging detector. In fact, every point in a sample is scaled up to an area with the diameter $d=l /(L / D)$ at the detector plane. This geometrical blurring effect was the dominant contribution in the tomography scans performed here on the final spatial resolution. A collimation ratio L/D of 250 was used with a $40 \mathrm{~mm}$ pinhole diameter.

The imaging camera used has a maximum exposure time of $30 \mathrm{~s}$ because it is not actively cooled. Although larger L/D ratios may usually be selected to improve the final spatial resolutions, this detector limitation prevented this possibility. That is, the fixed maximum exposure time set the maximum signal to noise ratio (SNR) achievable for each L/D configuration and neutron flux. The settings used were considered the best compromise between spatial resolution and SNR in the final radiographs. Low noise (actively cooled) imaging camera with longer exposure capabilities should overcome this issue which is a solution is currently under developments on the instrument.

A neutron beam size at sample position of $~ 70 \times 70 \mathrm{~mm}^{2}$ was used. Radiographs were collected using a CMOS neutron tomography camera [22] which had a $2048 \times 2048$ pixel sensor, with a sensitive area of $59.5 \times 59.5 \mathrm{~mm}^{2}$ at the neutron-sensitive scintillator screen. The scintillator screen $(50 \mu \mathrm{m}$ thick $6 \mathrm{LiF}: \mathrm{ZnS})$ "converts" neutrons into visible light while preserving their 2D distribution; the CMOS device images the emitted light through an objective lens system positioned at $90^{\circ}$ with respect to the neutron beam. For each measurement, multiple radiographs were acquired along with periodic dark 
and flat field images at an integration time of $30 \mathrm{~s}$ each. The dark field, $I_{\text {dark }}$, and flat field, $I_{0}$, images were used for normalization purpose to represent the attenuation of the beam instead of an intensity reading. The dark and flat field images are taken when the beam is off and on, respectively, while the sample is out of the beamline to account for camera noise and beam inhomogeneities. For each data type (sample, flat and dark field) an average of ten different radiographs were required to improve neutron statistics and noise reduction. The normalised image was calculated as shown in Equation (2).

$$
I_{\text {norm }}=\frac{I-I_{\text {dark }}}{I_{0}-I_{\text {dark }}}
$$

The full monoblock samples, see Figure $1 \mathrm{a}$ ) and b), were scanned in a $45^{\circ}$ tilt position to minimise the attenuation path length. Sample dimensions are shown in Table 3. The ROI samples were scanned for direct comparison with the X-ray tomography scans detailed in section 4.1. Because of the neutrons' relatively higher penetration it was possible to scan the ROI samples in the flat position i.e. $0^{\circ}$ tilt. Thus, all four samples (ITER_MB_ROI, ITER_HHFT_ROI, CCFE_MB, IPP_Wf-Cu) could be images in one scan by stacking them on top of each other. For the ROI samples, ITER_MB and CCFE_MB the number of projections taken were 707, 1204 and 1333 respectively. Each projection used the maximum acquisition time of $30 \mathrm{~s}$. Reconstruction of the $3 \mathrm{D}$ volume from $2 \mathrm{D}$ radiographs was completed with a standard filtered back-projection algorithm using Octopus Imaging Software (XRE NV, Gent, Belgium).

Table 3. Dimensions of the monoblock samples.

\begin{tabular}{lcccc}
\hline Sample & $\begin{array}{c}\text { Height } \\
(\mathbf{m m})\end{array}$ & $\begin{array}{c}\text { Width } \\
(\mathbf{m m})\end{array}$ & $\begin{array}{c}\text { Depth } \\
(\mathbf{m m})\end{array}$ & Material \\
\hline ITER_MB & 28 & 32 & 5.5 & W-Cu \\
CCFE_MB & 24 & 27 & 4 & W-Cu-Cu-CuCrZr \\
\hline
\end{tabular}

\subsection{Image processing}

CT data consists of a series of 2D image 'slices' comprising greyscale pixels, e.g. Figure 4. Each slice has a thickness attributed to it; often equal to the width and height of the 2D pixel, thus a 3D pixel (voxel) is formed. When the slices are stacked together these represent a 3D volume. All visualisation and analysis of the tomographic volumes was undertaken using the software Fiji [23]. This is a specific distribution of ImageJ [24] which includes plugins specifically to facilitate scientific image analysis.

It should be noted that interpretation of CT data is more subjective than scientific and relies on inherited knowledge through operator experience. The cause of observed features (indicated by a region of attenuation contrast) are attributed to the most likely factor considering the location, shape and greyscale values. For example, a small region of decreased greyscale voxels (as shown in Figure 18 ) is more likely to be a void rather than the inclusion of significantly less attenuating material that would have had to be introduced as contamination during manufacturing.

\section{Results}

Because the X-ray tomography used a cone beam source, moving the sample closer to the source magnifies the image on the detector which can be used to achieve smaller voxel widths. Whereas the neutron beamline at IMAT uses an approximately parallel beam which means that resultant voxel 
widths weren't sample dependent, but rather on the neutron setup of the pinhole and detector. Considering these factors, the voxel widths for each scan can be calculated, as shown in Table 4. It should be noted that the minimum resolvable feature size is not directly related to voxel widths alone because it is also a complex function of any intrinsic noise or scanning artefacts within the image. For the neutron CT the geometrical unsharpness, $d$, (also shown in Table 4) is quantifiable by the relationship between the maximum distance of the sample from the detector, I, and the L/D ratio.

Table 4. Resultant image qualities considering scanning parameters.

\begin{tabular}{lllll}
\hline Sample & $\begin{array}{c}\text { X-ray CT } \\
\text { Voxel width } \\
(\mu \mathrm{m})\end{array}$ & $\begin{array}{l}\text { Voxel width } \\
(\mu \mathrm{m})\end{array}$ & $\begin{array}{l}\text { Maximum distance, } \\
I(\mathrm{~mm})\end{array}$ & $\begin{array}{l}\text { Geometrical unsharpness, } \\
d(\mu \mathrm{m})\end{array}$ \\
\hline ITER_MB_ROI & 12.4 & 29 & 25 & 100 \\
ITER_HHFT_ROI & 11.7 & 29 & 25 & 100 \\
CCFE_MB_ROI & 10.9 & 29 & 25 & 100 \\
IPP_Wf-Cu & 12.6 & 29 & 22 & 88 \\
ITER_MB & N/A & 29 & 44 & 176 \\
CCFE_MB & N/A & 29 & 43 & 172 \\
\hline
\end{tabular}

Figure 4-Figure 7 are example cross sectional slices from the tomography data for the ROI samples. In each figure, the top row is a slice approximately midway along the pipe length (xy plane), the bottom row is a slice in the $x z$ plane. The left and right columns show data from the X-ray and neutron tomography data, respectively. Figure 8 shows example cross sections from the neutron tomography data sets for the full monoblock samples. The top and bottom rows show the $x y$ and $x z$ planes, respectively, and left and right columns show the ITER_MB and CCFE_MB samples, respectively.
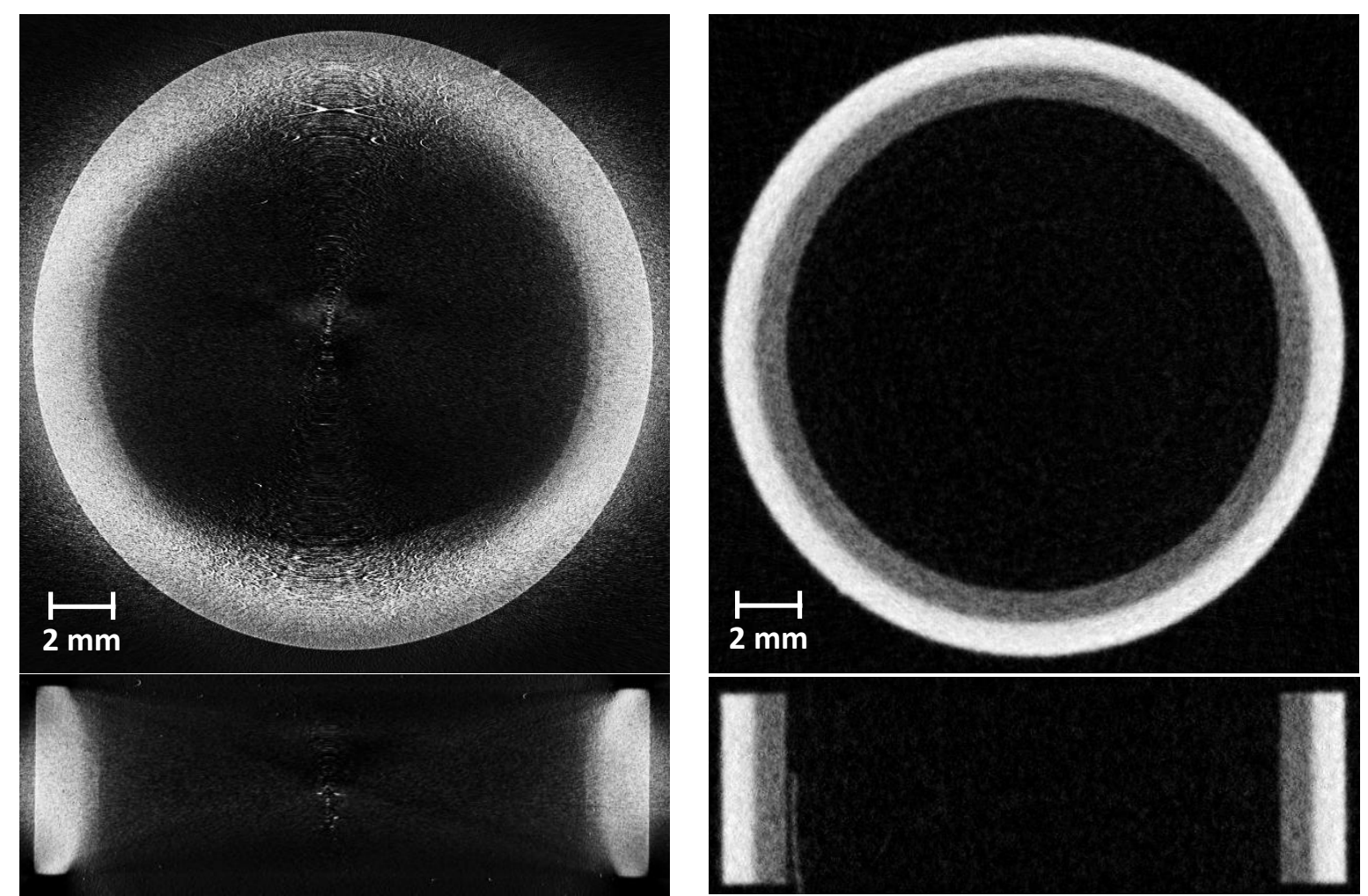

Figure 4. Example cross sectional tomography data slices from the $x y$ (top) and $x z$ (bottom) planes for the ITER_MB_ROI sample with a comparison of $X$-ray (left) vs. neutron (right) tomography imaging results. 

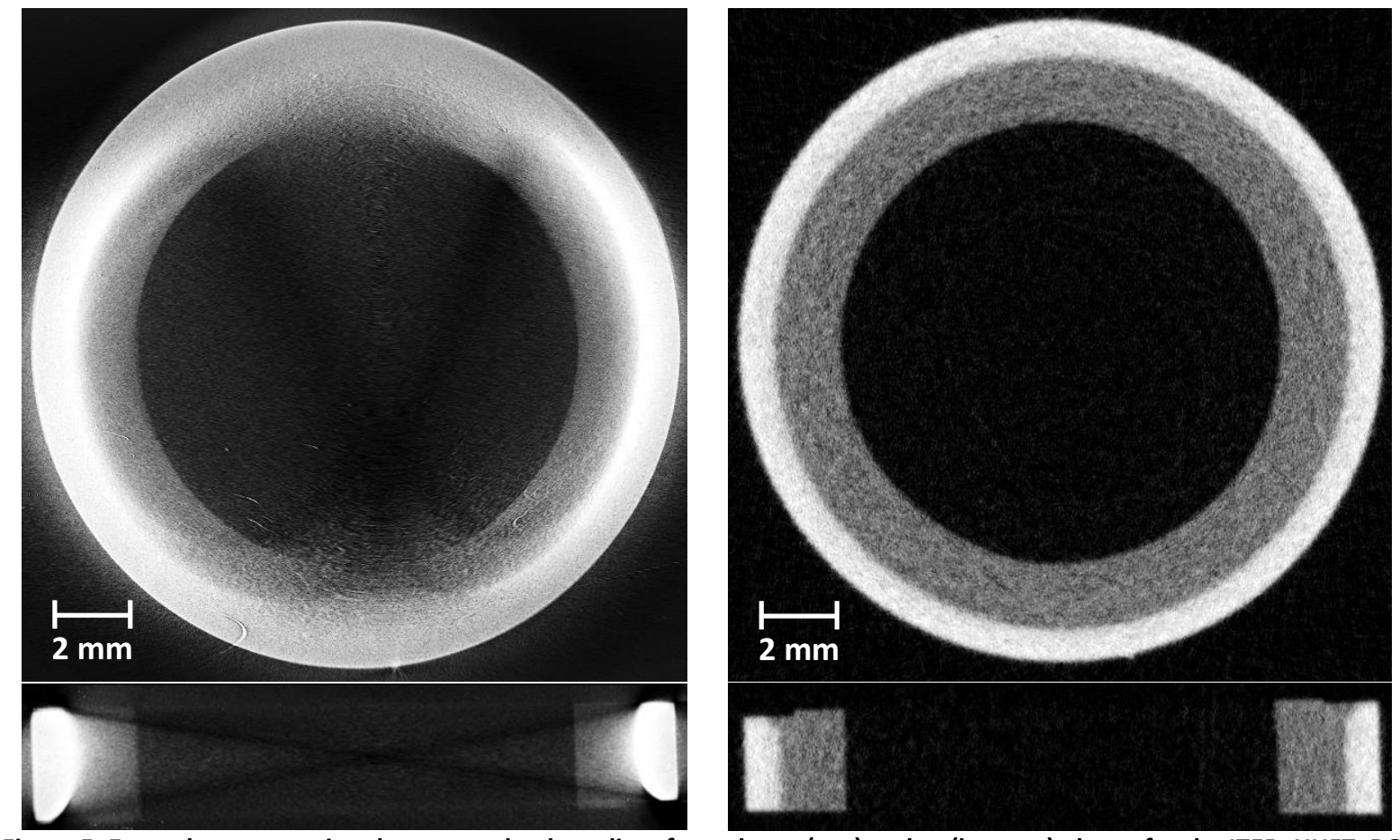

Figure 5. Example cross sectional tomography data slices from the $x y$ (top) and xz (bottom) planes for the ITER_HHFT_ROI sample with a comparison of X-ray (left) vs. neutron (right) tomography imaging results.
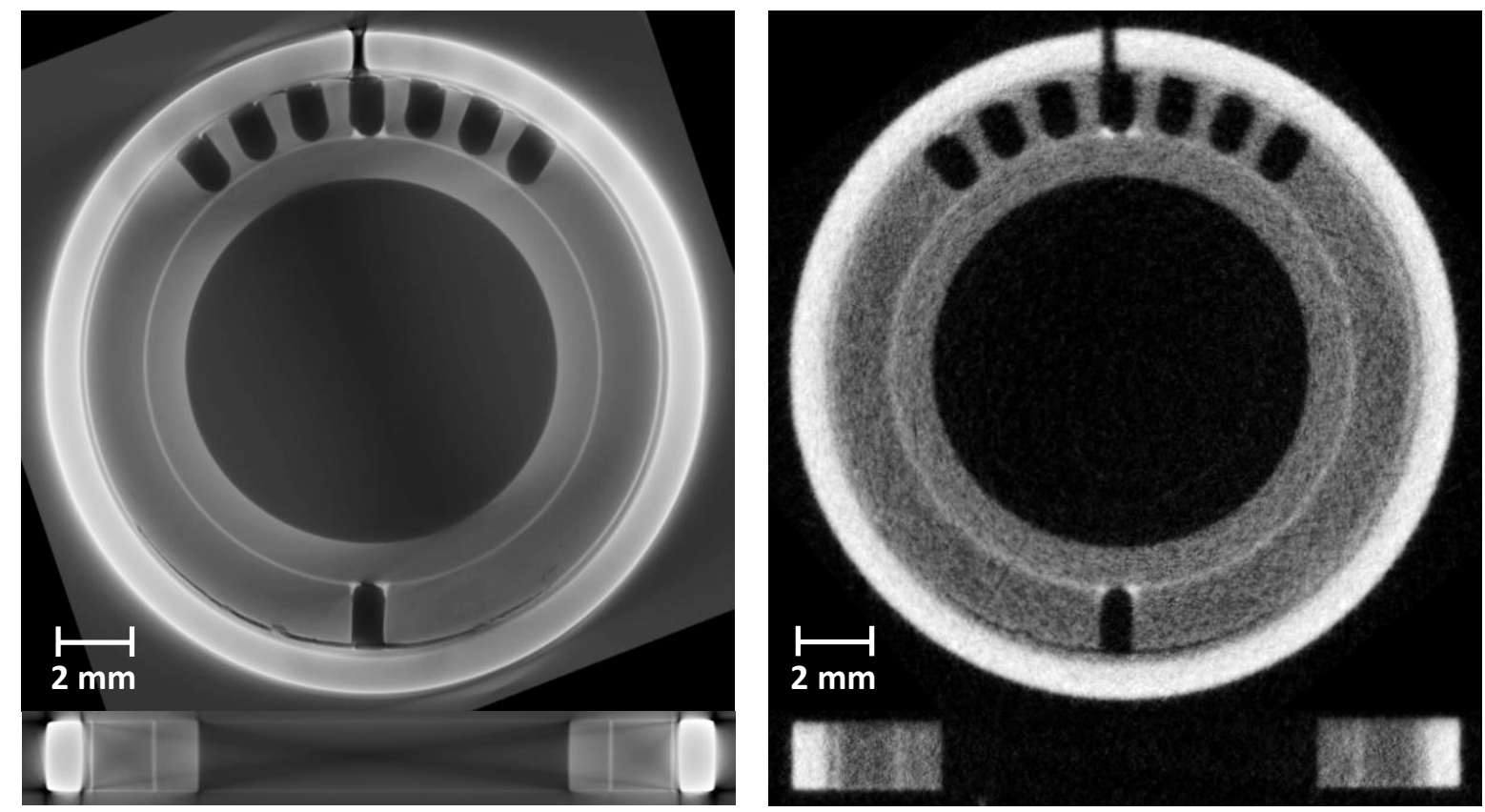

Figure 6. Example cross sectional tomography data slices from the $x y$ (top) and $x z$ (bottom) planes for the CCFE_MB_ROI sample with a comparison of X-ray (left) vs. neutron (right) tomography imaging results. 

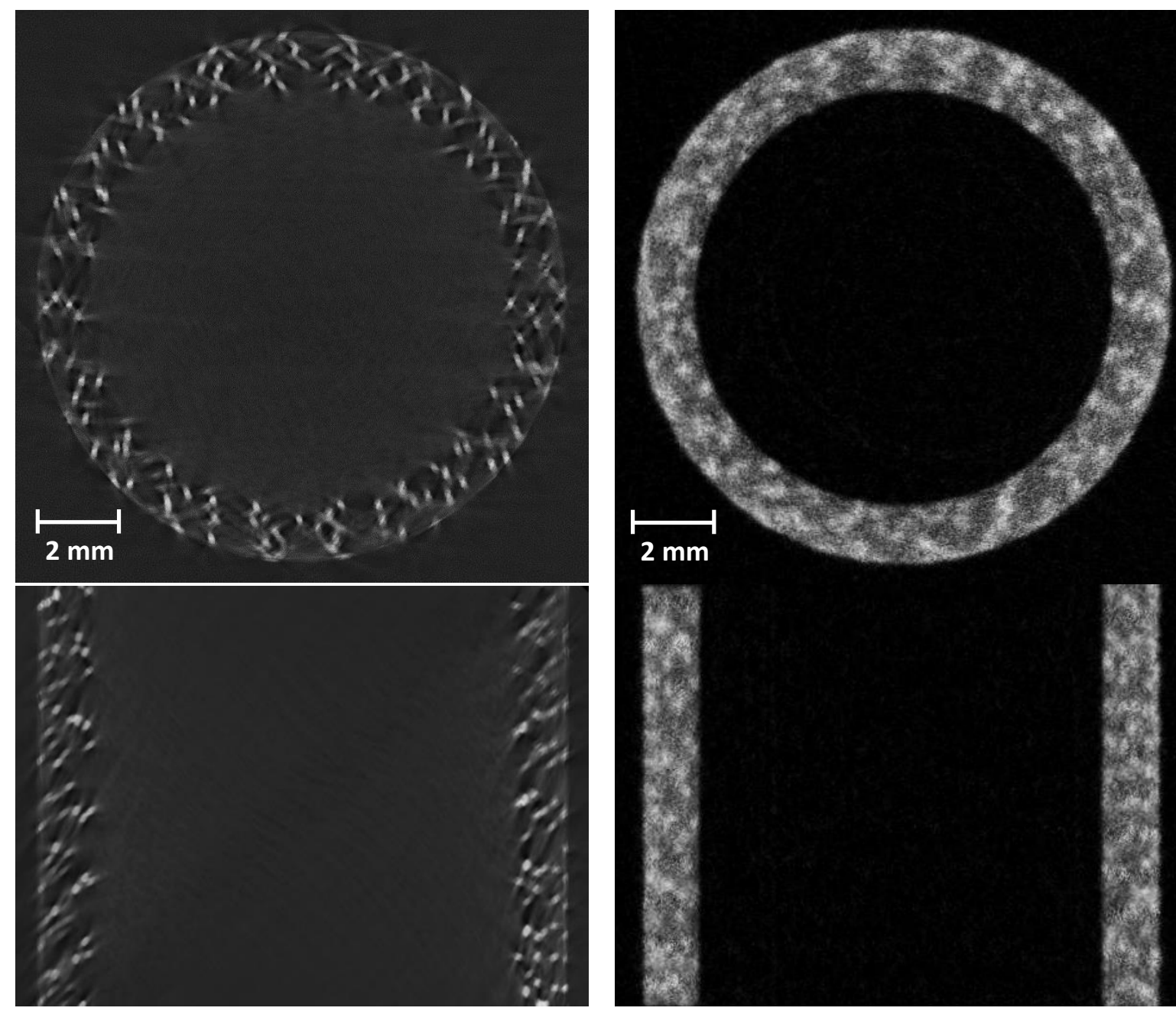

Figure 7. Example cross sectional tomography data slices from the $x y$ (top) and $x z$ (bottom) planes for the IPP_Wf-Cu sample with a comparison of X-ray (left) vs. neutron (right) tomography imaging results.
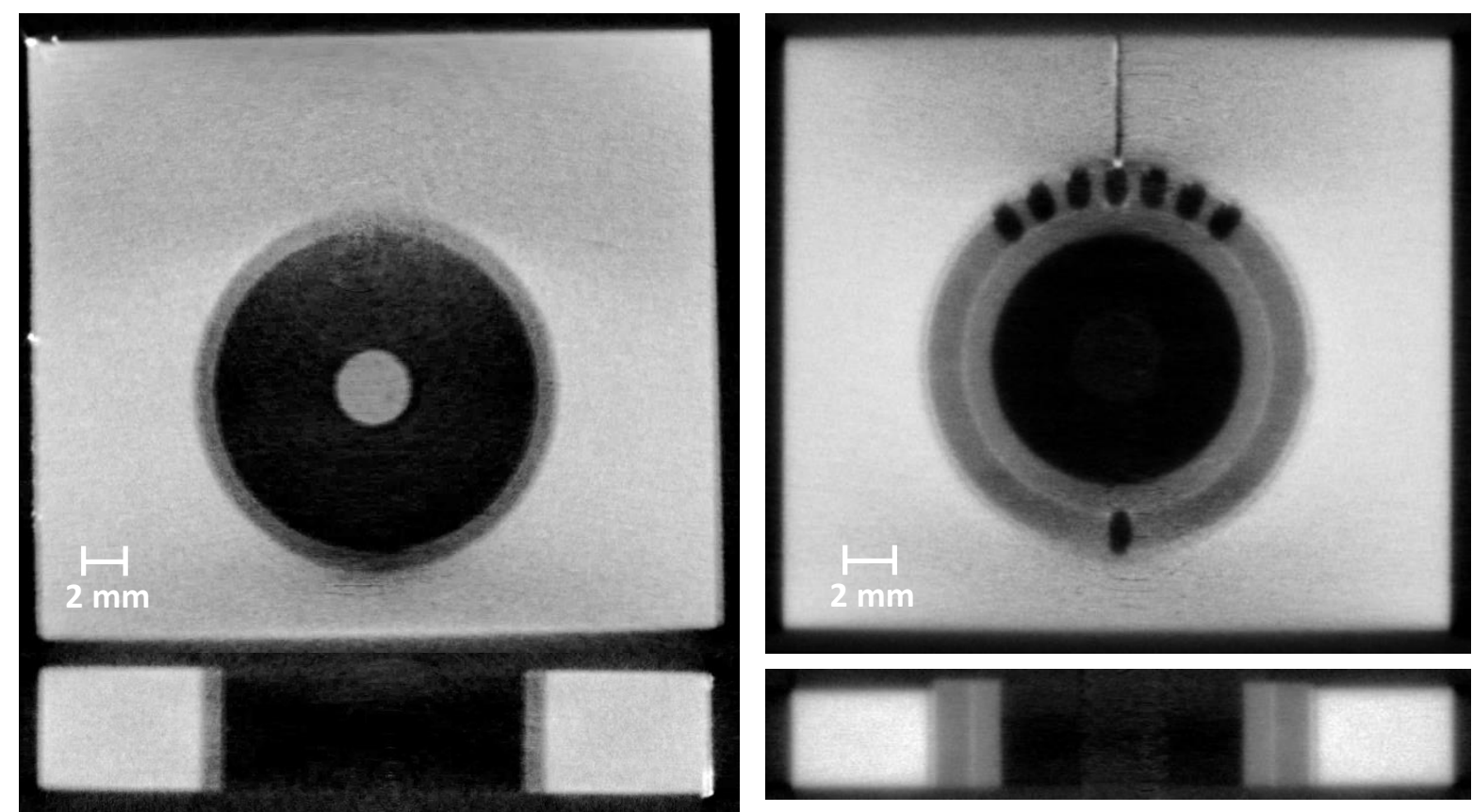

Figure 8. Example cross sectional neutron tomography data slices from the $x y$ (top) and xz (bottom) planes for the ITER_MB (left) and CCFE_MB (right) samples. 


\section{Discussion}

\subsection{Samples}

Firstly, is it worth considering the differences between samples and the potential impact on results. Using the sample dimensions, the total volumes and the volumes of the constituent parts were calculated, as shown in Table 5. The fibre volume fraction for the IPP_Wf-Cu sample was estimated to be $10 \%$, therefore the constituent part volumes are based on this assumption because they could not be calculated geometrically. Also included are values for X-ray attenuation path length (the 'chord' and 'max. path' columns) which are discussed in greater detail later in this section.

Table 5. Volume of samples and constituent parts as calculated geometrically.

\begin{tabular}{llllll}
\hline \multicolumn{1}{c}{ Sample } & \multicolumn{1}{c}{$\begin{array}{c}\text { Volume } \\
\left(\mathbf{m m}^{\mathbf{3}} \mathbf{)}\right.\end{array}$} & $\begin{array}{c}\text { W Volume } \\
\left(\mathbf{m m}^{\mathbf{3}} \mathbf{)}\right.\end{array}$ & $\begin{array}{c}\text { Cu Volume } \\
\left(\mathbf{m m}^{\mathbf{3}}\right)\end{array}$ & $\begin{array}{c}\text { Chord } \\
(\mathbf{m m})\end{array}$ & $\begin{array}{c}\text { Max. path } \\
(\mathbf{m m})\end{array}$ \\
\hline ITER_MB_ROI & 534 & 283 & 251 & 8.49 & 9.85 \\
ITER_HHFT_ROI & 342 & 78 & 264 & 5.74 & 6.48 \\
CCFE_MB_ROI & 352 & 55 & 297 & 5.92 & 6.24 \\
IPP_Wf-Cu & 542 & 54 & 488 & 8.31 & 13 \\
ITER_MB & 3956 & 3680 & 276 & & \\
CCFE_MB & 2278 & 1684 & 594 & & \\
\hline
\end{tabular}

+ Combined volume of pure $\mathrm{Cu}$ and Cu alloys.

The volume of material (particularly tungsten) is an important factor because higher levels of attenuation impacts ability to acquire 'clean' images. Comparison of the tungsten volume in the ROI samples shows that for ITER_MB_ROI it is 3.6 and 5.1 times greater than ITER_HHFT_ROI and CCFE_MB_ROI, respectively. However, the volume itself isn't a wholly accurate indicator of attenuation because a sample with evenly distributed attenuation paths across the detector is likely to be less challenging to image than a sample containing highly attenuating paths at particular angles. Thus, by considering the samples' maximum chord length, $c$, the maximum attenuation path through tungsten, $p$, was calculated, see Figure 9 and Table 5. Again, the value for ITER_MB_ROI is greater than the other two samples. The chord for ITER_HHFT_ROI is less than that of CCFE_MB_ROI, but because of the sample's length, the maximum attenuation path is therefore greater. Considering the volumes of tungsten and the maximum attenuation paths through tungsten it is predicted that ITER_MB_ROI will pose the greatest imaging challenge followed by ITER_HHFT_ROI, then CCFE_MB_ROI.

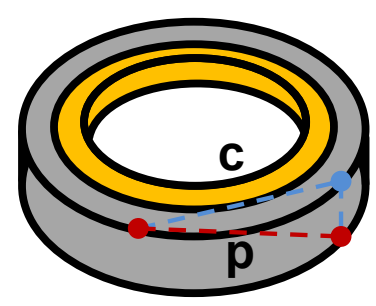

Figure 9. Schematic showing maximum chord length, c, through tungsten section of ROI samples and maximum attenuation path, $p$.

For IPP_Wf-Cu it is difficult to estimate the maximum attenuation path through tungsten because the precise location of the fibres is unknown. The chord length and maximum attenuation path have been included in Table 5 as indicative values, but the majority of this will be through copper. However, given the estimate of tungsten volume it is likely that the sample will pose a similar imaging challenge to that of CCFE_MB_ROI. 
Compared with the ROI samples, the full monoblocks (ITER_MB and CCFE_MB) contain significantly more tungsten than would have been possible to image with X-rays, which is why only neutrons were used. Here it can be seen that the ITER_MB contains significantly more tungsten than the CCFE_MB sample indicating that it poses the greatest imaging challenge.

\subsection{X-ray tomography}

The minimum resolvable feature of a tomography image is a result of the image's voxel widths (due to scanning parameters), the level of signal noise and reconstruction artefacts. In an ideal image it would require a minimum of three voxels to resolve a feature i.e. 0-1-0 for binarised data. In practice it typically requires 5-10 pixels. Following this rationale, features with a minimum size of 50-100 $\mu \mathrm{m}$ should be resolvable for the X-ray tomography, according to the voxel widths shown in Table 4 . When considering the ITER_MB_ROI sample, the presence of reconstruction artefacts is sufficiently significant that the W-Cu interface can't be distinguished (see Figure 4). Due to this, observation of interface features is not possible. Therefore, despite a $12.4 \mu \mathrm{m}$ voxel width, the minimum resolvable feature is orders of magnitude greater. In practice, this data is of no value for quantitative analysis and little value for qualitative analysis. Similarly, the same is true for ITER_HHFT_ROI (see Figure 5).

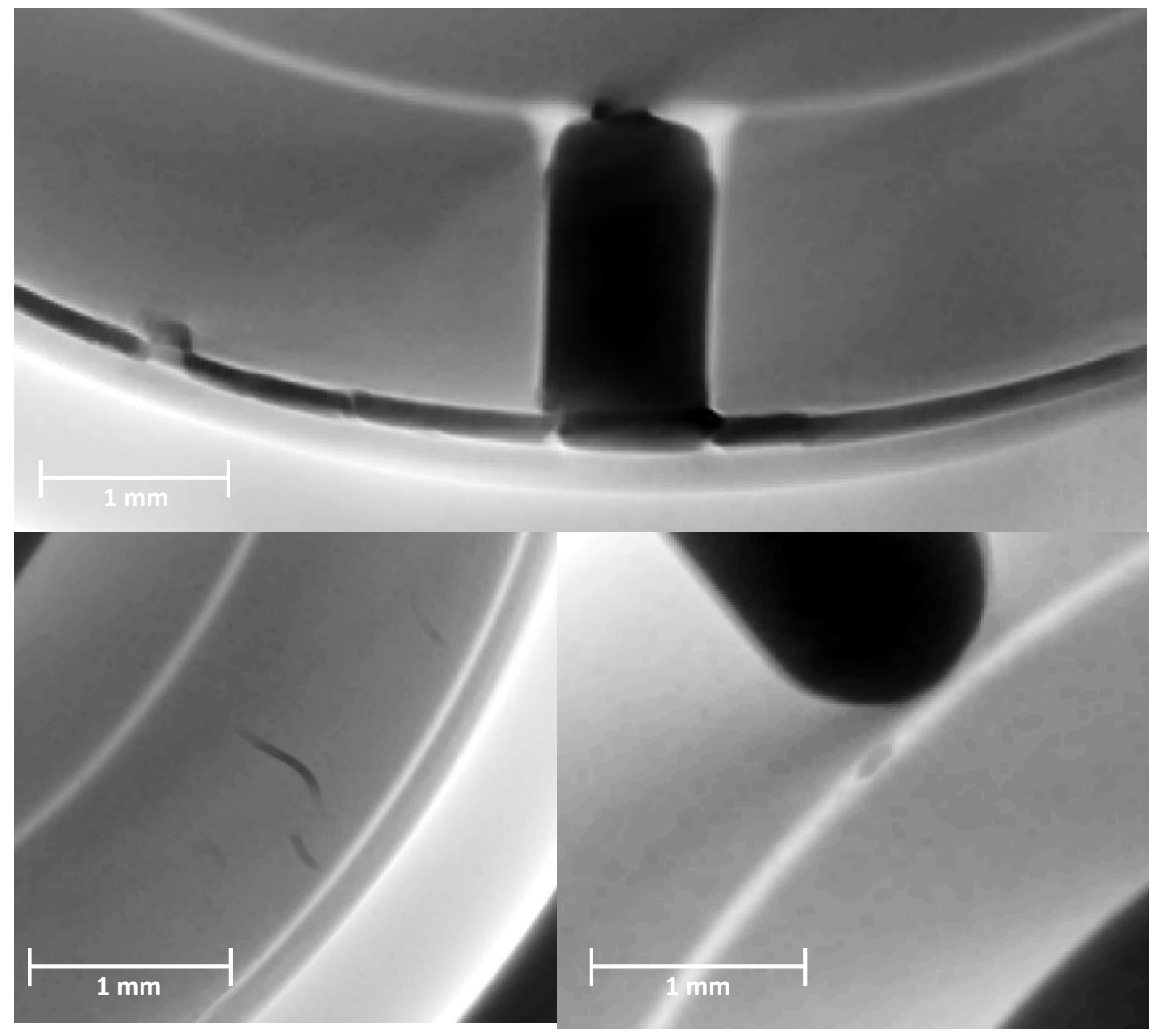

Figure 10. Zoomed in xy plane images from a X-ray tomography image of the CCFE_MB_ROI sample showing (top) debonding and variation in braze layer thickness, (bottom left) radial micro-cracking in $\mathrm{Cu}$ interlayer and (bottom right) pore within braze layer. 
The CCFE_MB_ROI sample has much improved definition such that the W-Cu interface is clearly seen. This is due to the reduced $\mathrm{X}$-ray attenuation path through $\mathrm{W}$. This confirms the prediction made in section '6.1 Samples'. Observable features on the external surfaces of the CCFE_MB_ROI sample, such as braze layer thickness, were compared with measurements made by optical microscopy. By this method it was ascertained that that features of the order of $60 \mu \mathrm{m}$ were resolvable. That is, features greater in size than $\sim 6$ voxels can be observed, confirming the previous assumption. Each layer of the sample is distinguishable, from inside to out. That is $\mathrm{CuCrZr}$ coolant pipe; braze; $\mathrm{Cu}$ interlayer; braze; cast Cu layer; $W$ armour. Examples of internal features of observable interest are shown in .

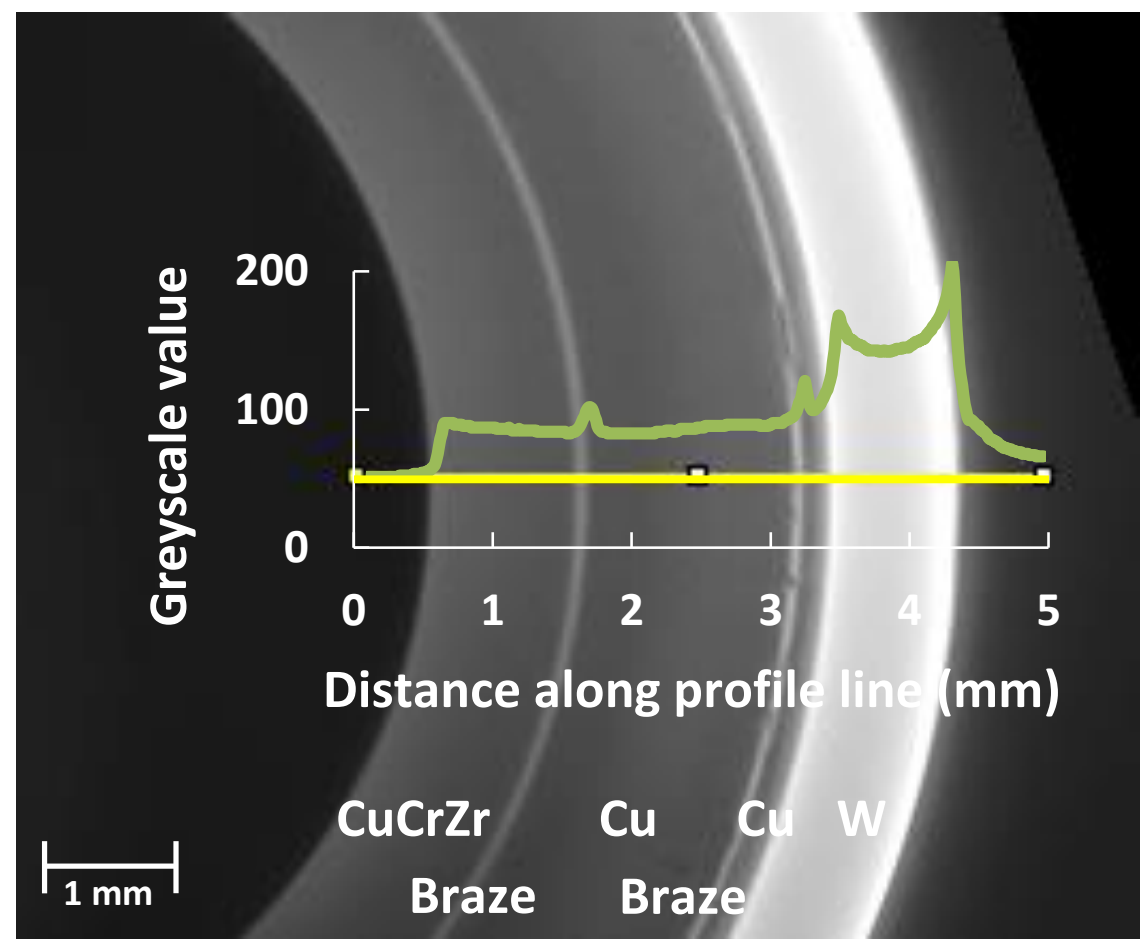

Figure 11. Plot of greyscale values (green) with respect to distance along profile line (yellow). Plot is overlaid on tomographic slice where profile was taken to show direct relation between changes in greyscale and image features.

Quantification of greyscale variation through the constituent was done by taking a profile line through a central tomography slice, shown in Figure 11. Peaks are observed at the $\mathrm{CuCrZr-Cu}$ and $\mathrm{Cu}-\mathrm{Cu}$ interfaces and at the $\mathrm{W}$ layer edges. The peaks at the $\mathrm{Cu}$ interfaces are due to the higher attenuation of Au in the braze filler material. The peak widths are representative of the $75 \mu \mathrm{m}$ thick braze material. The peaks at the $W$ edges are caused by the well-known phenomenon of beam hardening. This occurs when polychromatic beams are used and the soft X-rays (lower energy) are filtered by the sample giving the false appearance of a change in attenuation through the sample. That is, the change in greyscale through the $\mathrm{W}$ is not caused by a change in the material (e.g. density) but rather an artefact of the imaging technique. This should be taken into consideration when interpreting the images as it can lead to overestimating layer thicknesses. It is also worth noting that the contrast in greyscale levels of the $\mathrm{CuCrZr}$ and $\mathrm{Cu}$ layers is very low. Therefore, without the presence of the more highly attenuating braze at the interface it might be challenging to identify features in this region.

Figure 12 shows the xz plane where edge streaking artefacts are clearly observed, in particular from the $W$ armour. Streaking artefacts typically happen when undersampling occurs, explaining its prominence around the $\mathrm{W}$ armour. On the edges this has a blurring effect on the higher greyscale 
levels, increasing the difficult to accurately distinguish the material boundary, as seen in the gradual decrease of greyscale level in the profile in Figure 11. At the corners, the opposite is true, where the artefact causes the corners to appear 'filleted' or 'smoothed off'. Both these imaging effects must be considered when attempting to make quantitative metrology measurements of the sample. The other parts of the sample can be seen to be less affected.

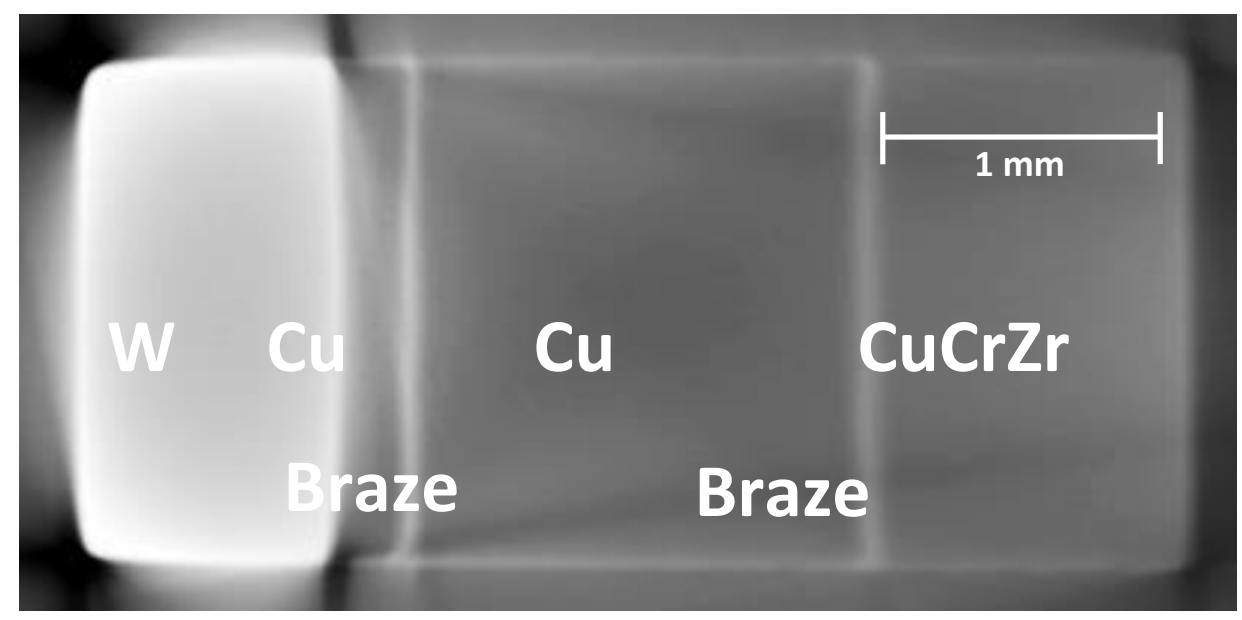

Figure 12. Zoomed in xz plane image from a X-ray tomography image of the CCFE_MB_ROI sample.

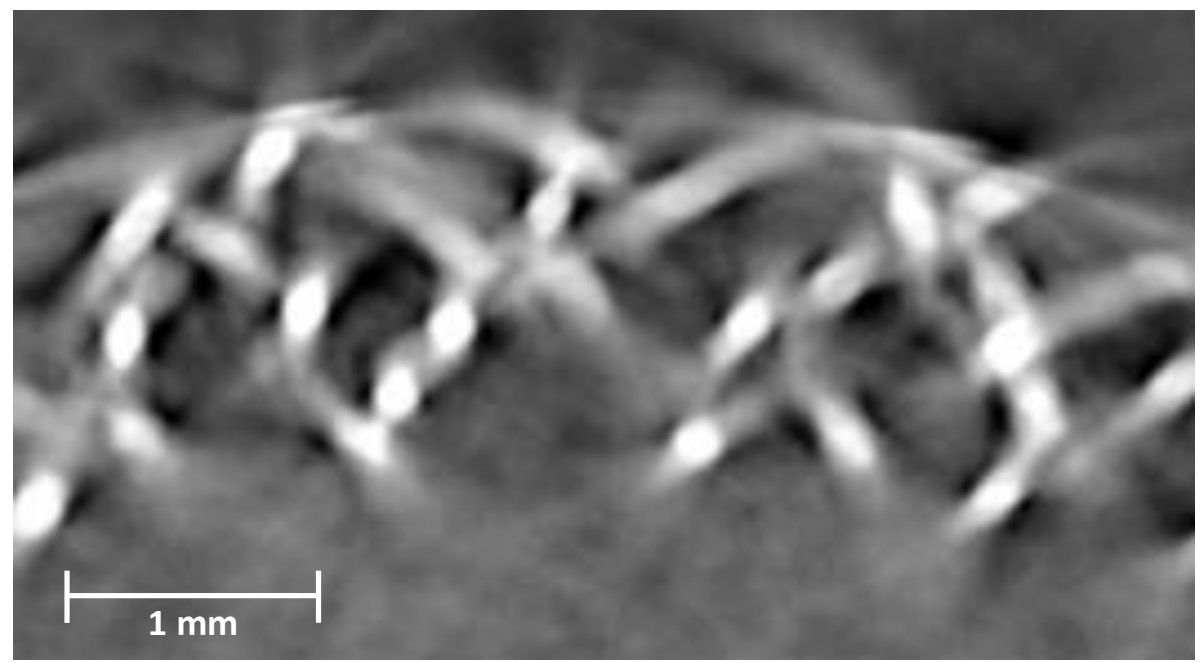

Figure 13. Zoomed in xy plane image from X-ray tomography image of IPP_Wf-Cu sample demonstrating that individual fibres are resolvable to locate positioning. Also shown is a lack of contrast between $\mathrm{Cu}$ matrix and background air; also streaking artefacts from $\mathrm{W}$ fibres are visible.

The 'quality' of the resulting image from the scan of the IPP_Wf-Cu sample (see Figure 7) was an improvement over the ITER_MB_ROI and ITER_HHFT_ROI samples but not as clear as the CCFE_MB_ROI sample. The resulting voxel width of this scan was $12.6 \mu \mathrm{m}$ which was sufficient to distinguish the individual composite fibres which had a diameter of $150 \mu \mathrm{m}$. However, the high signal attenuation contrast between the $\mathrm{W}$ fibres and Cu matrix created a particular challenge in capturing detail within the $\mathrm{Cu}$ phase of the composite. It can be seen that there is low contrast in greyscale values between the $\mathrm{Cu}$ matrix and the surrounding background air which means that the sample boundaries are not well defined. In addition to this, streaking artefacts are prominent from each of the $\mathrm{W}$ fibres (see Figure 13). To quantify the variation in greyscale through the constituent parts of the sample a profile line through a central tomography slice is shown in Figure 14. In addition to 
demonstrating the similarity in greyscale values of $\mathrm{Cu}$ and background phases it can be seen that the streaking artefacts in fact cause zones within the $\mathrm{Cu}$ matrix to appear less attenuating than the background, as shown by the troughs in the plot.

In practicality, these artefacts makes it challenging investigate micro-features at the fibre-matrix interface or perform metrology quantifications. Despite this, clear fibre definition means that the centreline of each fibre can easily be found and visualised to investigate deviation of fibre placement from the ideal weave pattern. Figure 15 is a 3D visualisation of the fibre placements which can be viewed interactively using the post-processing software. Visualising the image along radial slices allows investigation of the weave pattern in the cylindrical coordinate space as shown in Figure 16.

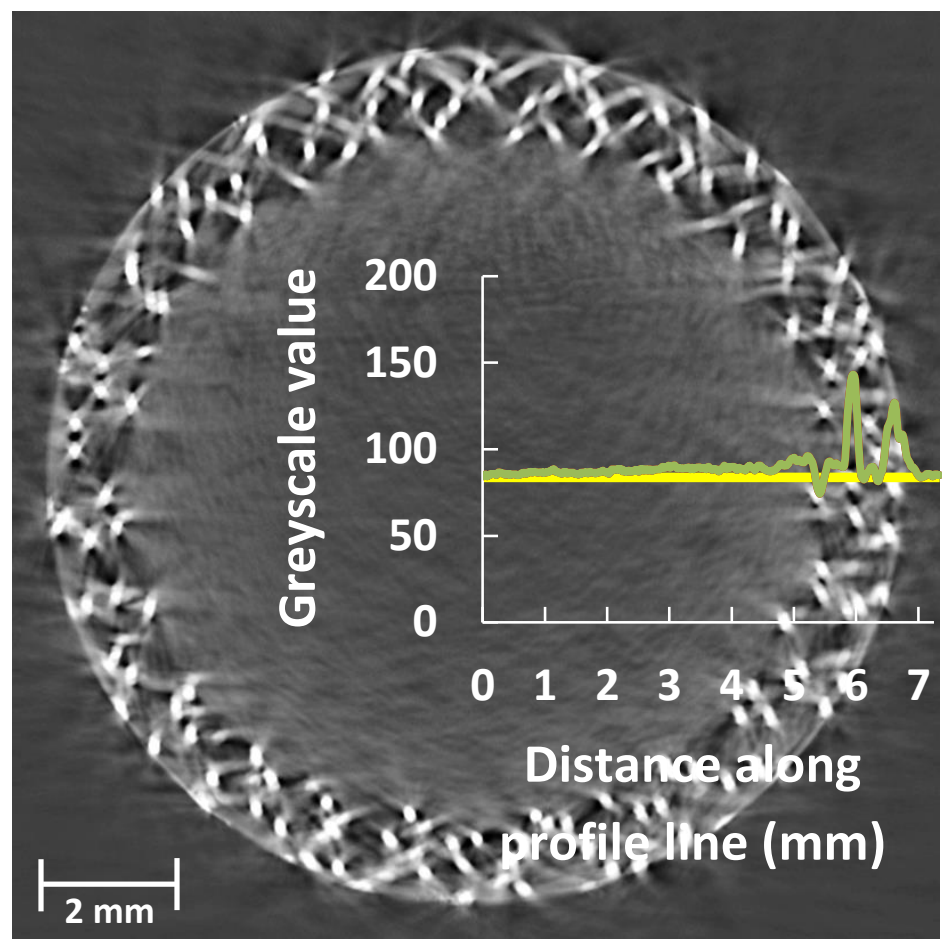

Figure 14. Plot of greyscale values (green) with respect to distance along profile line (yellow). Plot is overlaid on X-ray tomographic slice where profile was taken to show direct relation between changes in greyscale and image features.

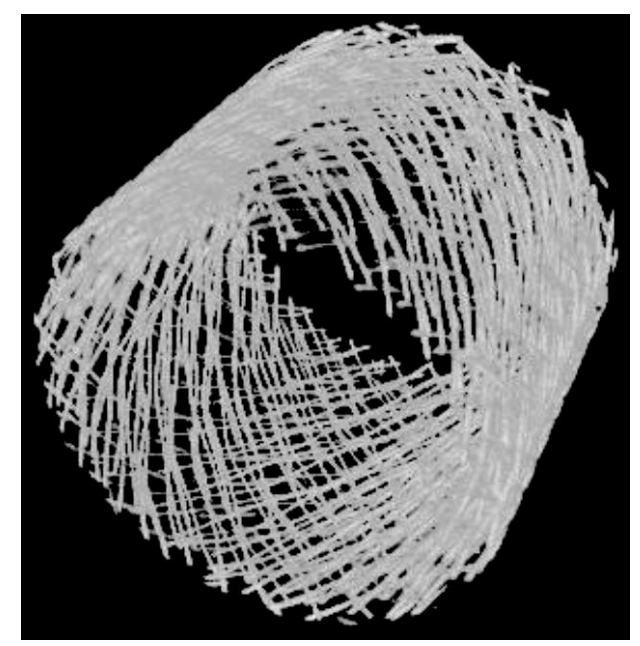

Figure 15. 3D visualisation from $\mathrm{X}$-ray tomography data of $\mathrm{W}$ fibre placement within IPP_Wf-Cu sample, Cu matrix has been made transparent to assist viewing. 


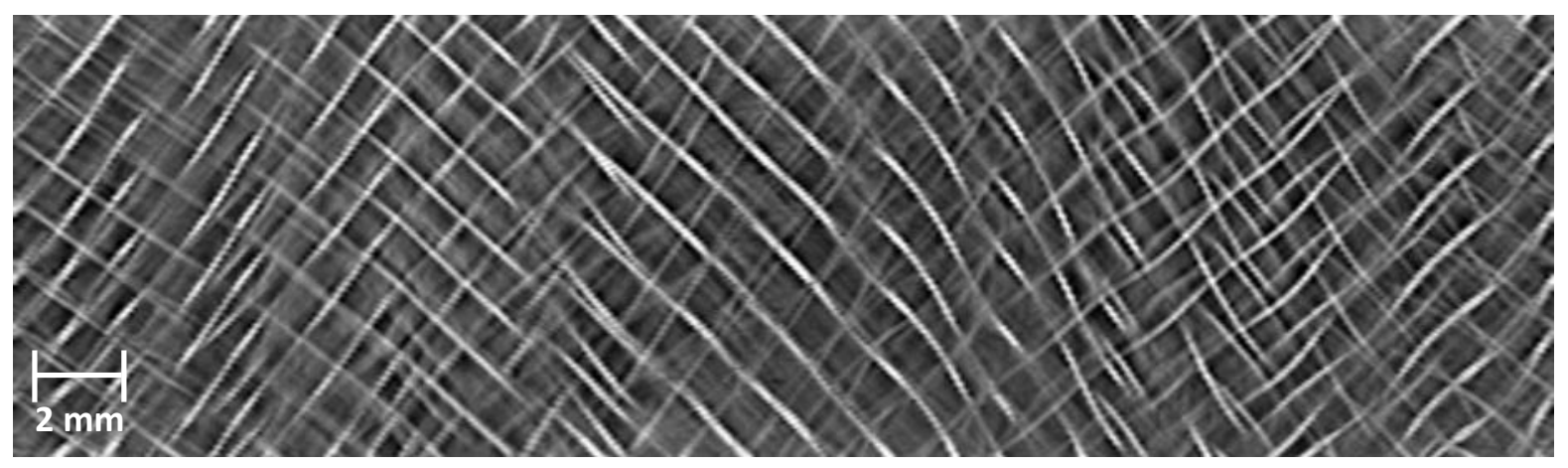

Figure 16. 'Unrolled' section from X-ray tomography image of IPP_Wf-Cu sample at radius $=5.75 \mathrm{~mm}$ (i.e. $x$-axis is along $\theta$ and $y$-axis is along length of pipe).

\subsection{Neutron tomography}

For the scanning parameters were identical for all images collected with neutron tomography. To characterise the performance of the imaging system a 'Siemens star' test pattern placed in closecontact with the detector was used to measure the modulation transfer function (MTF) [25]. A radiograph was taken, see, using identical with scanning parameters. The MTF indicated that the imaging system would be capable of resolving features greater than $112 \mu \mathrm{m}$. This is smaller than the geometrical unsharpness, Table 4, and therefore could not be further improved experimentally.
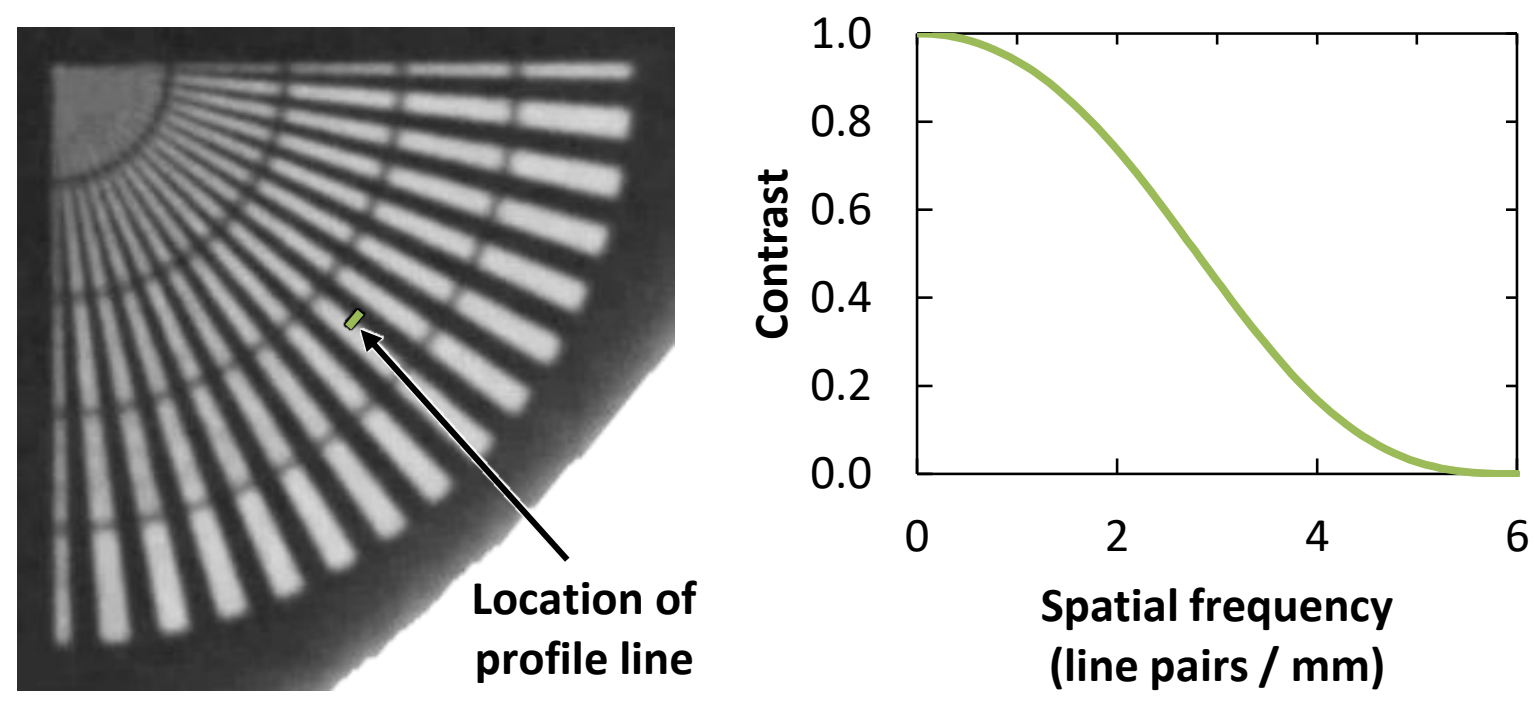

Figure 17. (a) Neutron radiograph of 'Siemens star' and location of profile line (green) used to calculate modulation transfer function. (b) Plot showing contrast with respect to spatial frequency. This curve is used to calculate the modulation transfer function and thus infer the theoretical minimum resolvable feature.

The main reason for use of neutron tomography was to investigate imaging of full monoblocks nondestructively. This was successfully demonstrated, see Figure 8, on both ITER_MB and CCFE_MB. Comparison with optical microscopy showed that surface features greater than $250 \mu \mathrm{m}$ were resolvable. Similarly sized features were observed internally, for example a void found in the braze layer of CCFE_MB as shown in Figure 18 and Figure 19. The image resolution impeded measurement of the braze layer thickness of $75 \mu \mathrm{m}$, however, it was clearly visible at the layer interfaces. It should also be noted that the neutron imaging also displayed a contrast in greyscale level between the $\mathrm{CuCrZr}$ of the inner coolant pipe and the $\mathrm{Cu}$ of the functional interlayer (see Figure 18). Very little contrast was observed in the X-ray tomography image. For the ITER_MB sample there were no features of 
interest visible at the W-Cu interface indicating a good join due to no defect being present or that they were smaller than the imaging resolution. An interesting observation was that the sample's serial ID which had been the laser etched to a relatively shallow depth on an external surface, see Figure 20, was observable. The etching appears brighter in the image (i.e. more highly attenuating) than bulk W. It is possible that this is either a product of a change in the $\mathrm{W}$ microstructure or due to material contamination during the etching process. In general, the level of noise and scanning artefacts was low enough to perform qualitative observations and quantitative measurements.

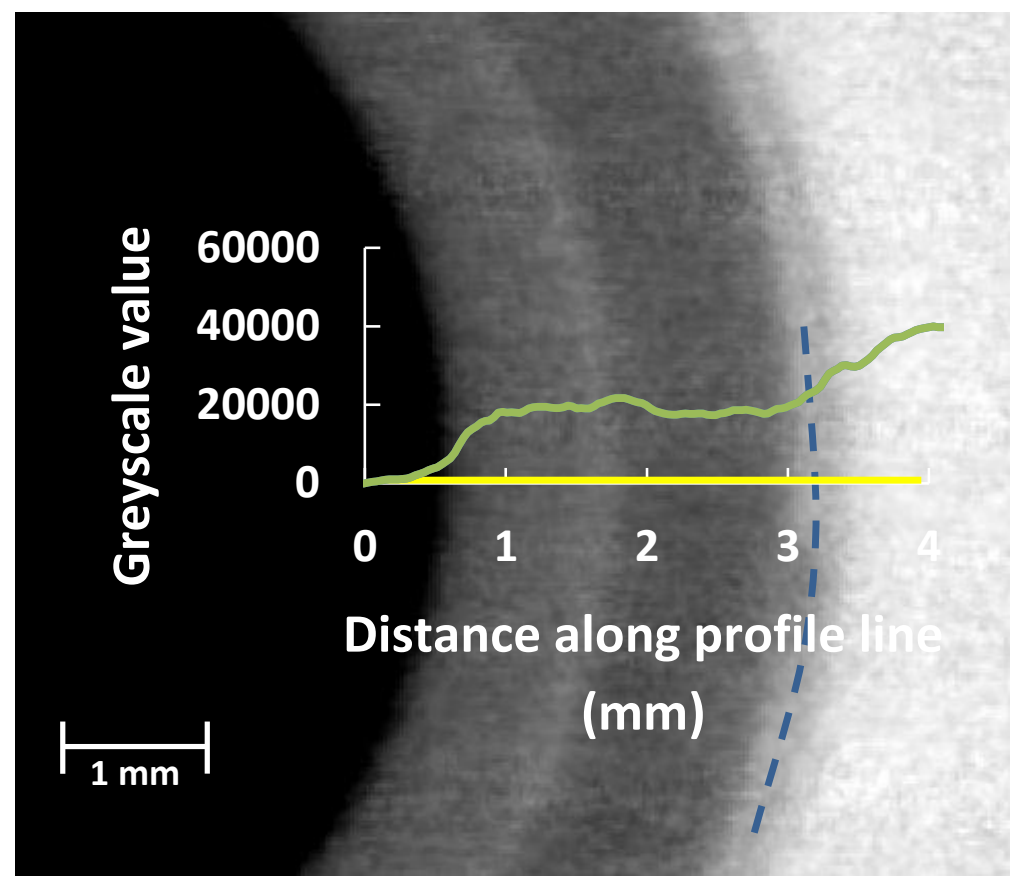

Figure 18. Plot of greyscale values (green) with respect to distance along profile line (yellow). Plot is overlaid on neutron tomographic slice where profile was taken to show direct relation between changes in greyscale and image features. Also shown is (dashed blue) profile line used for Figure 19 to highlight void observed in braze layer.

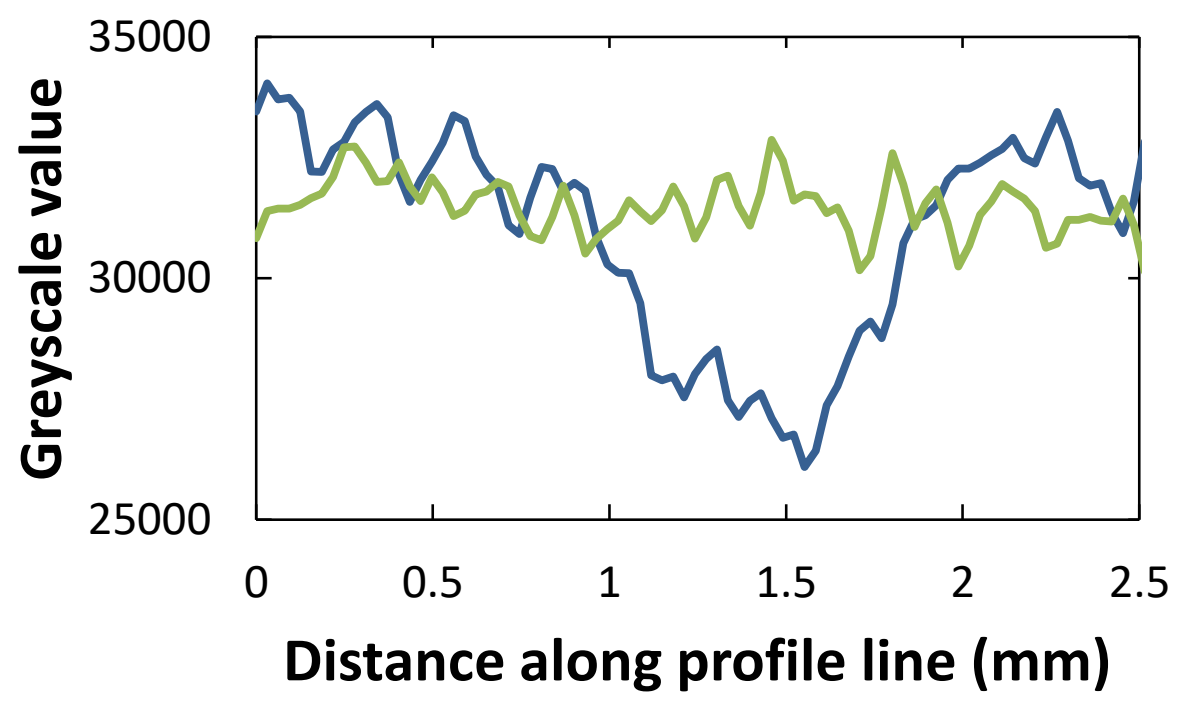

Figure 19. Plot of greyscale values with respect to distance along (dashed blue) profile line from Figure 18. Reduction in greyscale value $1.5 \mathrm{~mm}$ along profile line shows location of void in braze layer. For comparison, a second (green) profile line from the same sector but at a greater depth shows the typical variation in greyscale due to image noise. 


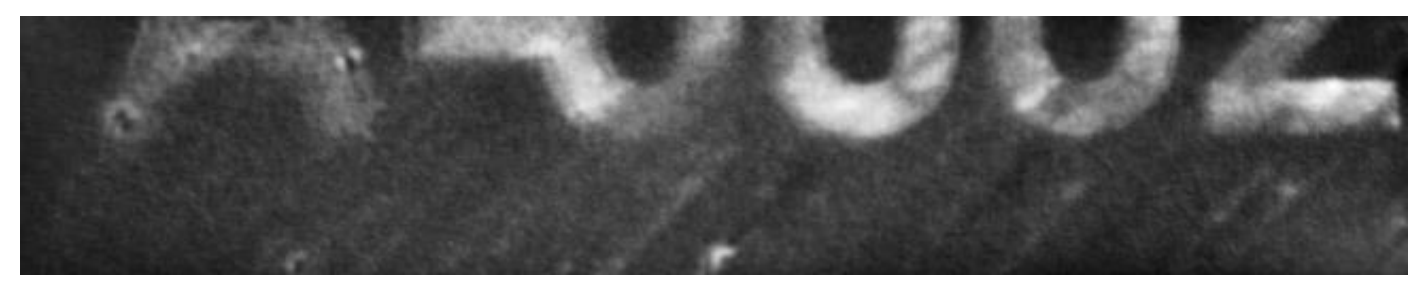

Figure 20. Part of laser etched serial ID on outer surface of ITER_MB sample that was visible in neutron tomography image.

In addition to the full monoblock samples, the ROI samples and IPP_Wf-Cu were imaged by neutron tomography for direct comparison with X-ray tomography. All of the samples were successfully scanned with images resolving features of similar size to that of the full MB samples. The images of ITER_MB_ROI and ITER_HHFT_ROI (see Figure 4 and Figure 5, respectively) show a clear interface between the $\mathrm{W}$ armour and $\mathrm{Cu}$ interlayer. There were no observable features at the $\mathrm{W}$-Cu interface indicating either that none were present or that they were smaller in size than the spatial resolution. There was no observable contrast between the $\mathrm{Cu}$ and $\mathrm{CuCrZr}$ layers in the ITER_HHFT_ROI sample. Because there was observable contrast in the CCFE sample, this suggests that there exists some compositional difference in either the $\mathrm{Cu}$ or $\mathrm{CuCrZr}$ materials between the ITER and CCFE samples. The $\mathrm{Cu}-\mathrm{CuCrZr}$ join in the ITER_HHFT_ROI sample was performed via hot radial pressing and therefore did not require any filler (braze) material. Subsequently, no markers were present to identify the $\mathrm{Cu}$ $\mathrm{CuCrZr}$ interface. As the location of the interface was known, by measuring the radial distance it was possible to investigate this region which was found not to contain any observable features (i.e. none were present, or they were smaller than the image resolution). When observing the ITER_HHFT_ROI sample in the xz plane it was found that there was a bevel (or chamfer) in the W-Cu interface near the top of the sample (see Figure 22). This is likely to be a by-product of the manufacturing process.

For the CCFE_MB_ROI sample (see Figure 6) the features observable were similar to that of the CCFE_MB samples. That is, the braze layer and some defects within this could be seen but the resolution wasn't sufficient to measure the braze thickness. This was expected due to identical scanning parameters being used. There was a small reduction in image noise for the CCFE_MB_ROI sample. Figure 21 shows a comparison of a similar region in the two samples. Again a contrast in greyscale levels was observed between the $\mathrm{Cu}$ and $\mathrm{CuCrZr}$ layers.

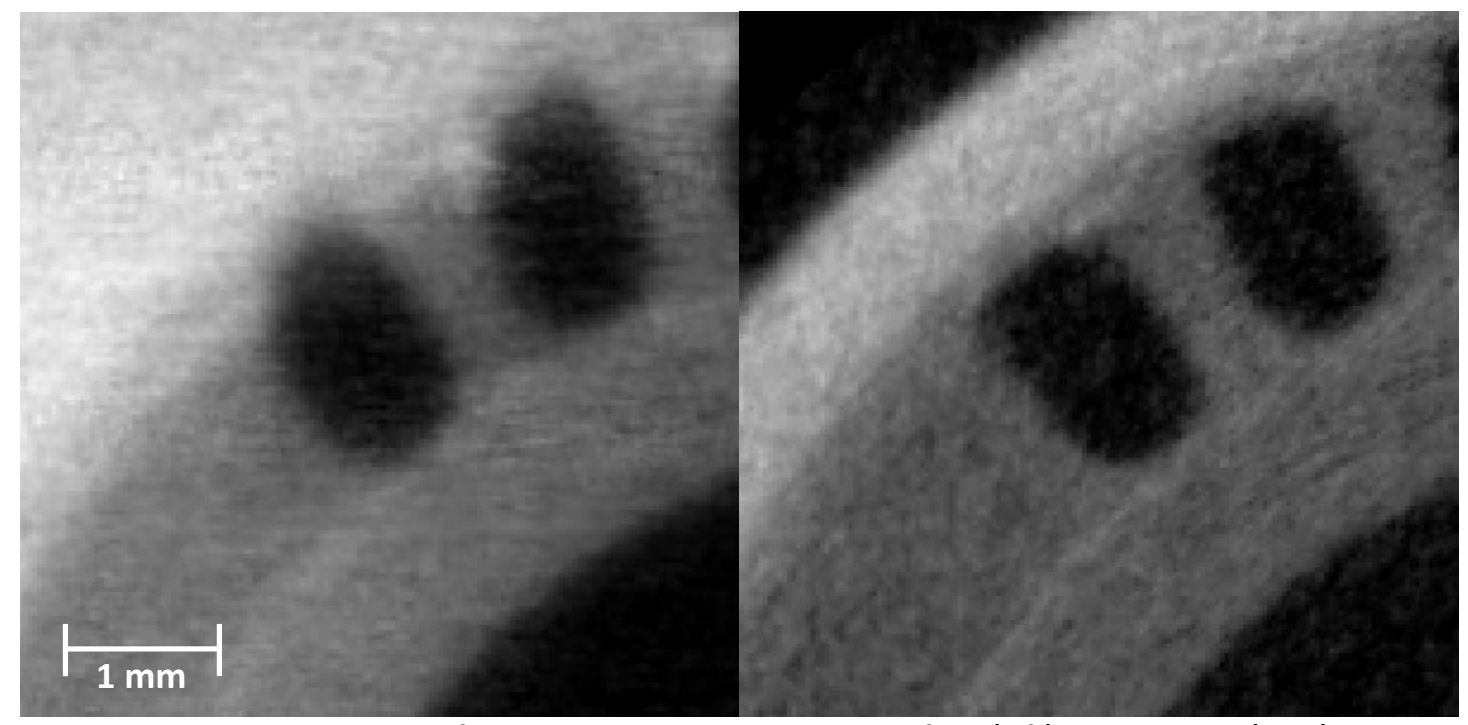

Figure 21. Zoomed in xy plane images from neutron tomography images of the (left) CCFE_MB and (right) CCFE_MB_ROI samples for comparison. 
The $150 \mu \mathrm{m}$ diameter of the $\mathrm{W}$ fibres in the IPP_Wf-Cu was greater than the geometric unsharpness of $88 \mu \mathrm{m}$ meaning that it was expected that the fibres would be observable, as is indeed the case. In the xy plane, crossing the fibre diameter, defining the fibre-matrix boundary is challenging because of the image noise (see Figure 23). However, in the radial direction the definition is clearly visible (see Figure 24) and can be used to investigate fibre placement (see Figure 25). It should be noted that, although noise is present, there is distinct contrast in the greyscale levels of the background air, $\mathrm{Cu}$ matrix and $\mathrm{W}$ fibres which was not present in the $\mathrm{X}$-ray tomography image. Additionally, there are no streaking artefacts emitting from the $W$ fibre edges. This image is sufficient for qualitative observation of the fibres but does not have adequate resolution to investigate the fibre-matrix interface.

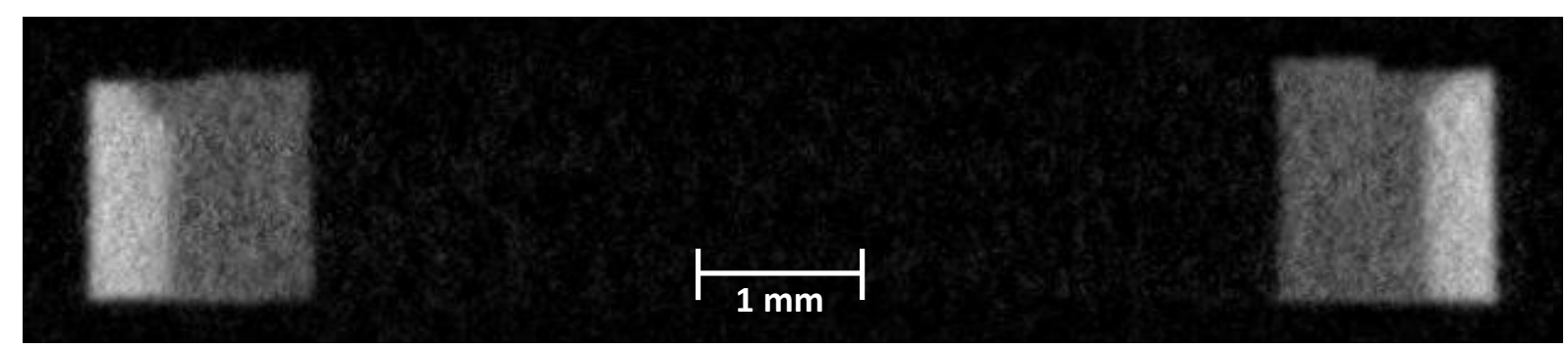

Figure 22. Cross sectional neutron tomography slices from the xz plane for the ITER_HHFT_ROI sample.

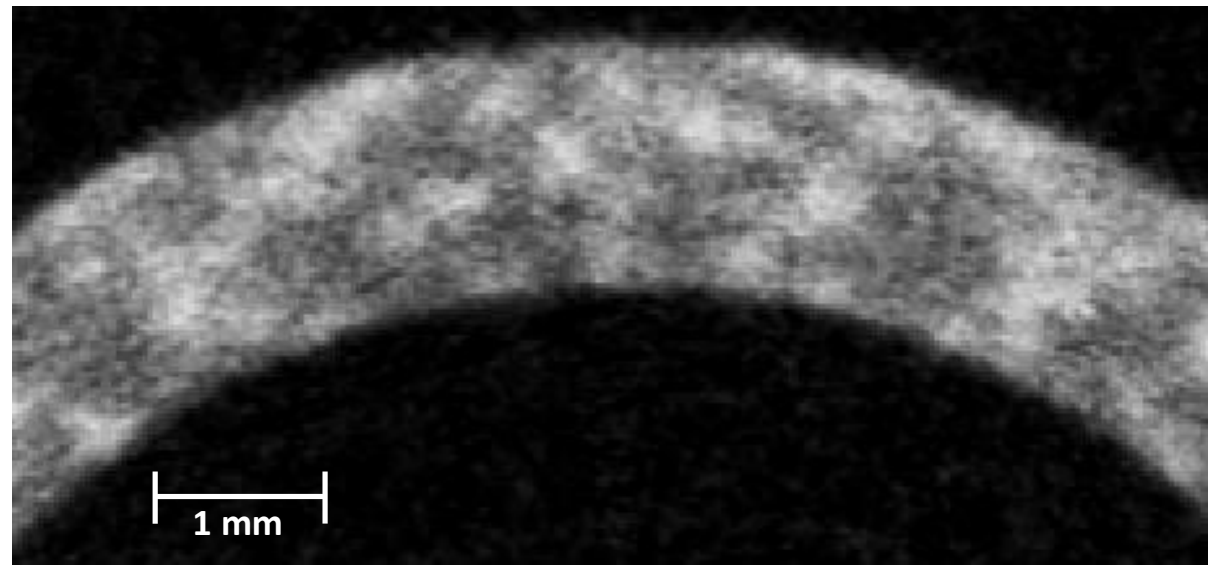

Figure 23. Zoomed in xy plane image from neutron tomography image of IPP_Wf-Cu sample.

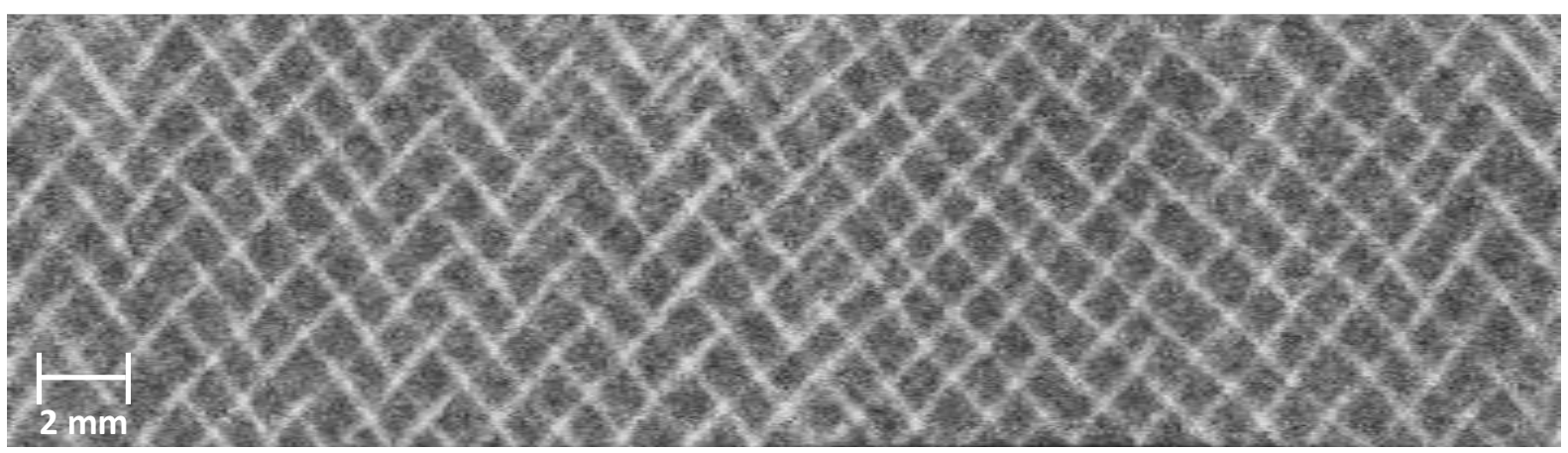

Figure 24. 'Unrolled' section from neutron tomography image of IPP_Wf-Cu sample at radius $=5.75 \mathrm{~mm}$ (i.e. $x$-axis is along $\theta$ and $y$-axis is along length of pipe). 


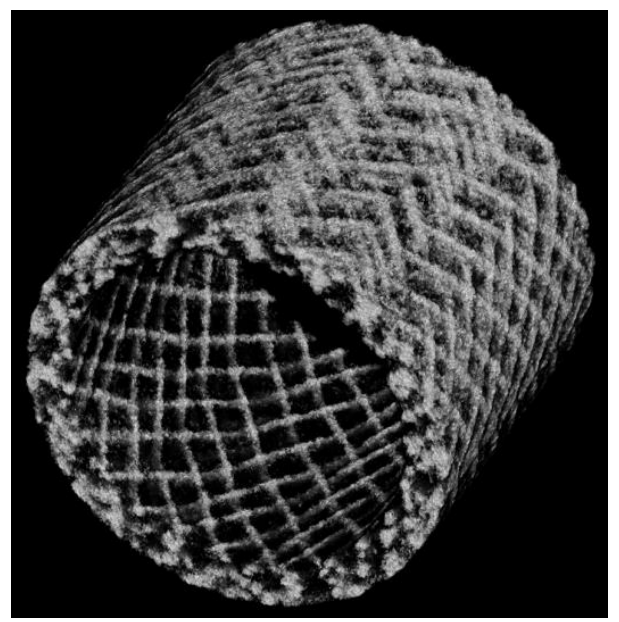

Figure 25. 3D visualisation from neutron tomography data of $\mathrm{W}$ fibre placement within IPP_Wf-Cu sample, Cu matrix has been made transparent to assist viewing.

\subsection{Comparison of $\mathrm{X}$-ray and neutron tomography data}

Despite $\mathrm{W}$ being a relatively high attenuator of $\mathrm{X}$-rays and neutrons, both tomography methods were shown capable of imaging samples containing $\mathrm{W}$, albeit with varying degrees of success.

A few key logistical aspects differentiate the techniques. Because X-ray tomography is less penetrating of $\mathrm{W}$ it was required to machine the MBs into $\mathrm{ROI}$ samples. An obvious disadvantage of this approach is that it is no longer a non-destructive testing (NDT) method. If to be used during manufacturing production, this would restrict its use to batch sampling. Comparatively, neutron tomography of full MB was possible which means it may be used as an NDT method.

X-ray tomography equipment is increasingly becoming an industry standard technique for NDT, therefore equipment is readily available and comparatively cheap. However, neutron tomography is currently only available at select facilities globally which impacts operational costs. The current cost of purchasing an X-ray CT scanner is approximately equivalent to 50-100 days of neutron tomography operational costs as a service. The low availability and increased cost of neutron tomography may be prohibitive in scanning large numbers of MBs. However, demonstrated here was the ability to scan multiple samples in one neutron tomography scan when stacked upon each other. This would be appropriate for scanning monoblock assemblies rather than individual samples. Another consideration when using neutron tomography is that samples become activated through interaction with the neutrons. The amount of waiting time required before samples may then be transported depends on the length of time the sample is left in the neutron beam. For the scans in this study this was of the order of weeks, but the level of activity is low and samples may be handled manually.

For the ROI samples, the ITER_MB_ROI and ITER_HHFT_ROI X-ray tomography images contained such a high level of artefacts that they were unusable. The increase in the maximum attenuation path through these samples was $60 \%$ and $4 \%$, respectively, compared with the CCFE_MB_ROI sample. The thickness of the W layer in both the ITER_HHFT_ROI and CCFE_MB_ROI samples was $0.5 \mathrm{~mm}$, but the total W volume was greater for the ITER_HHFT_ROI sample. It is therefore apparent that the quality of the resultant X-ray tomography image is highly sensitive to the amount of tungsten in the sample due to its high level of $X$-ray attenuation. If a sufficient volume of $W$ is removed it is possible to obtain a valuable 3D image via $X$-ray tomography. 
By directly comparing the $\mathrm{X}$-ray and neutron tomography images for the CCFE_MB_ROI sample it is clear that the $\mathrm{X}$-ray image provided valuable additional resolution and less noise. Smaller features were observable and those visible in both methods could more accurately be quantitatively measured with X-ray tomography. However, this was the only sample where this was proven true meaning if $\mathrm{X}$ ray tomography was to be used provisions must be made to reduce the $X$-ray attenuation path through $W$. The neutron tomography was shown to provide adequate image detail to perform qualitative observations for all samples. Therefore, both scanning techniques are useful but with different benefits. It would be more appropriate to use the higher resolution X-ray tomography for the research and development cycle where samples may be tested destructively and neutron tomography for quality assurance of manufactured assemblies.

Recently hardware developments have seen the introduction of higher energy X-ray tomography scanners [26]. It is possible that these would allow increased length of attenuation paths through W. This is likely to be a modest increase that would still require the production of ROI samples.

\section{Conclusions}

A comparative study was performed using X-ray and neutron tomography to investigate the appropriateness of the techniques to inspect the manufacturing of a heat exchanger component for a fusion energy device. It was demonstrated that both techniques were capable of imaging components which included tungsten which is a relatively high attenuator of X-rays and neutrons. Each technique had its own benefits and drawbacks.

X-ray tomography was shown to be highly sensitive to the length of the maximum attenuation path through tungsten. Thus, it was required to machine region of interest samples from larger components for investigations. In this instance, investigations focussed on the quality of the joining interface between the inner $\mathrm{Cu}$ alloy coolant pipe and outer $\mathrm{W}$ armour. In samples where sufficient tungsten was removed the resulting image had relatively high resolution (features of $60 \mu \mathrm{m}$ or greater were observable) and value for qualitative and quantitative analysis. Where insufficient tungsten was removed images were effectively unusable.

Due to the differences in the neutron tomography technique, images will have a lower baseline resolution than what is possible with X-ray tomography. However, the reason for using neutron tomography is for when X-ray tomography is not possible due to large signal attenuation e.g. full components. As such, the main goal for using neutron tomography was to demonstrate the feasibility of using the technique as a non-destructive method (i.e. removing the need to produce 'region of interest' samples). This was successfully demonstrated on full heat exchanger components (divertor monoblock). For a direct comparison with the X-ray tomography, the 'region of interest' samples were also imaged. As expected, the resulting resolution was lower that $\mathrm{X}$-ray tomography ranging from 88 $\mu \mathrm{m}$ to $176 \mu \mathrm{m}$ for the IPP_Wf-Cu and ITER_MB samples, respectively. However, they could be used non-destructively and yielded images adequate for quality assurance. It is believed that this is the first time that a full tungsten divertor monoblock has been imaged by computerised tomography.

Insufficient samples were tested to draw conclusions on the candidate designs shown here. This work was a proof of concept that both these tomography methods can produce valuable data either for the research and development cycle of fusion component design or in quality assurance of manufacturing if used appropriately. 


\section{Acknowledgements}

This work has been carried out within the framework of the EUROfusion Consortium and has received funding from the Euratom research and training programme 2014-2018 under grant agreement No 633053, from the RCUK Energy Programme [grant number EP/1501045] and EPSRC [grant number EP/R012091/1]. To obtain further information on the data and models underlying this paper please contact PublicationsManager@ccfe.ac.uk. The views and opinions expressed herein do not necessarily reflect those of the European Commission.

\section{References}

[1] A. S. Kukushkin, H. D. Pacher, V. Kotov, G. W. Pacher, and D. Reiter, "Finalizing the ITER divertor design: The key role of SOLPS modeling," Fusion Eng. Des., vol. 86, no. 12, pp. 2865-2873, 2011.

[2] M. Merola and G. Vieider, "On the use of flat tile armour in high heat flux components," J. Nucl. Mater., vol. 258-263, pp. 672-676, Oct. 1998.

[3] J. H. You et al., "Conceptual design studies for the European DEMO divertor: Rationale and first results," Fusion Eng. Des., vol. 109-111, no. PartB, pp. 1598-1603, 2016.

[4] R. A. Pitts et al., "A full tungsten divertor for ITER: Physics issues and design status," J. Nucl. Mater., vol. 438, pp. S48-S56, Jul. 2013.

[5] Y. Zhang, A. Galloway, J. Wood, M. B. O. Robbie, D. Easton, and W. Zhu, "Interfacial metallurgy study of brazed joints between tungsten and fusion related materials for divertor design," J. Nucl. Mater., vol. 454, no. 1-3, pp. 207-216, Nov. 2014.

[6] S. Cao, C. Somsen, M. Croitoru, D. Schryvers, and G. Eggeler, "Focused ion beam/scanning electron microscopy tomography and conventional transmission electron microscopy assessment of Ni4Ti3 morphology in compression-aged Ni-rich Ni-Ti single crystals," Scr. Mater., vol. 62, no. 6, pp. 399-402, Mar. 2010.

[7] S. Roccella et al., "Non-destructive methods for the defect detection in the ITER high heat flux components," Fusion Eng. Des., vol. 86, no. 9-11, pp. 1791-1796, Oct. 2011.

[8] M. Richou, A. Durocher, M. Medrano, R. Martinez-Oña, J. Moysan, and B. Riccardi, "Data merging of infrared and ultrasonic images for plasma facing components inspection," Fusion Eng. Des., vol. 84, no. 7-11, pp. 1593-1597, Jun. 2009.

[9] LI. M. Evans et al., "Thermal characterisation of ceramic/metal joining techniques for fusion applications using X-ray tomography," Fusion Eng. Des., vol. 89, pp. 826-836, 2014.

[10] S. E. Pryse and L. Kersley, "A preliminary experimental test of ionospheric tomography," J. Atmos. Terr. Phys., vol. 54, no. 7-8, pp. 1007-1012, Jul. 1992.

[11] U. Hampel, “6 - X-ray computed tomography," in Industrial Tomography, 2015, pp. 175-196.

[12] A. Zivelonghi, T. Weitkamp, and A. Larrue, "Open porosity and 3D pore architecture in plasmasprayed tungsten," Scr. Mater., vol. 115, pp. 66-70, Apr. 2016.

[13] M. Strobl, I. Manke, N. Kardjilov, A. Hilger, M. Dawson, and J. Banhart, "Advances in neutron radiography and tomography," J. Phys. D. Appl. Phys., vol. 42, no. 24, p. 243001, Dec. 2009.

[14] M. Fursdon et al., "The development and testing of the thermal break divertor monoblock target design delivering $20 \mathrm{MW}$ m -2 heat load capability," Phys. Scr., vol. T170, no. T170, p. 
14042, Dec. 2017.

[15] A. v. Muller et al., "Melt infiltrated tungsten-copper composites as advanced heat sink materials for plasma facing components of future nuclear fusion devices," Fusion Eng. Des., vol. 124, pp. 455-459, Nov. 2017.

[16] A. C. Kak and M. Slaney, Principles of Computerized Tomographic Imaging. Society for Industrial and Applied Mathematics, 2001.

[17] British Standard, "Non destructive testing - Radiation methods - Computed tomography - Part 2: Principle, equipment and samples," pp. 1-21, 2011.

[18] British Standard, "Non destructive testing - Radiation methods - Computed Tomography Part 3: Operation and interpretation," no. September 2011, 2011.

[19] T. Minniti et al., "Materials analysis opportunities on the new neutron imaging facility IMAT@ISIS," J. Instrum., vol. 11, no. 3, pp. C03014-C03014, Mar. 2016.

[20] W. Kockelmann et al., "Status of the Neutron Imaging and Diffraction Instrument IMAT," in Physics Procedia, 2015, vol. 69, pp. 71-78.

[21] T. Minniti, K. Watanabe, G. Burca, D. E. Pooley, and W. Kockelmann, "Characterization of the new neutron imaging and materials science facility IMAT," Nucl. Instruments Methods Phys. Res. Sect. A Accel. Spectrometers, Detect. Assoc. Equip., vol. 888, pp. 184-195, Apr. 2018.

[22] V. Finocchiaro, F. Aliotta, D. Tresoldi, R. C. Ponterio, C. S. Vasi, and G. Salvato, "The autofocusing system of the IMAT neutron camera," Rev. Sci. Instrum., vol. 84, no. 9, p. 93701, Sep. 2013.

[23] J. Schindelin et al., "Fiji: an open-source platform for biological-image analysis.," Nat. Methods, vol. 9, no. 7, pp. 676-82, Jun. 2012.

[24] J. Schindelin, C. T. Rueden, M. C. Hiner, and K. W. Eliceiri, "The ImageJ ecosystem: An open platform for biomedical image analysis," Mol. Reprod. Dev., vol. 82, no. 7-8, pp. 518-529, Jul. 2015.

[25] E. L. Nickoloff and R. Riley, "A simplified approach for modulation transfer function determinations in computed tomography," Med. Phys., vol. 12, no. 4, pp. 437-442, Jul. 1985.

[26] H. Villarraga-Gómez, "Seeing is Believing: X-ray Computed Tomography for Quality Control," Quality, vol. 55, no. 6, pp. 21-23, 2016. 